\title{
Substrate access mechanism in a novel membrane-bound phospholipase A of Pseudomonas aeruginosa concordant with specificity and regioselectivity
}

\author{
Sabahuddin Ahmad ${ }^{1}$, Christoph Heinrich Strunk ${ }^{2}$, Stephan N. Schott- \\ Verdugo ${ }^{1,3,5}$, Karl-Erich Jaeger ${ }^{2,4}$, Filip Kovacic ${ }^{2, *}$, Holger Gohlke ${ }^{1,5, *}$
}

1. Institute for Pharmaceutical and Medicinal Chemistry, Heinrich Heine University Düsseldorf, 40225 Düsseldorf, Germany

2. Institute of Molecular Enzyme Technology, Heinrich Heine University Düsseldorf, Forschungszentrum Jülich $\mathrm{GmbH}, 52425$ Jülich, Germany

3. Centro de Bioinformática y Simulación Molecular (CBSM), Faculty of Engineering, University of Talca, 3460000 Talca, Chile

4. Institute of Bio- and Geosciences (IBG-1: Biotechnology), Forschungszentrum Jülich $\mathrm{GmbH}, 52425$ Jülich, Germany

5. John von Neumann Institute for Computing (NIC), Jülich Supercomputing Centre (JSC), Institute of Biological Information Processing (IBI-7: Structural Biochemistry) \& Institute of Bio- and Geosciences (IBG-4: Bioinformatics), Forschungszentrum Jülich $\mathrm{GmbH}, 52425$ Jülich, Germany

${ }^{*}$ Corresponding authors

Address correspondence to: Prof. Dr. Holger Gohlke, Institute for Pharmaceutical and Medicinal Chemistry, Heinrich Heine University Düsseldorf, Düsseldorf, Germany; John von Neumann Institute for Computing (NIC), Jülich Supercomputing Centre (JSC), Institute of Biological Information Processing (IBI-7: Structural Biochemistry) \& Institute of Bio- and Geosciences (IBG-4: Bioinformatics), Forschungszentrum Jülich $\mathrm{GmbH}, 52425$ Jülich, Germany; Phone: (+49) 21181 13662; Email: gohlke@uni-duesseldorf.de, h.gohlke@fz-juelich.de and Dr. Filip Kovacic, Institute of Molecular Enzyme Technology, Heinrich Heine University Düsseldorf, Forschungszentrum Jülich GmbH, 52425 Jülich, Germany; Phone: (+49) 246161 2947; Email: f.kovacic@fz-juelich.de

Running title: Substrate access in PlaF

Keywords: membrane protein, phospholipid, mutagenesis, molecular dynamics simulations, free energy computations 


\section{Abstract}

PlaF is a cytoplasmic membrane-bound phospholipase $A_{1}$ from Pseudomonas aeruginosa that alters the membrane glycerophospholipid (GPL) composition and fosters the virulence of this human pathogen. PlaF activity is regulated by a dimer-tomonomer transition followed by tilting of the monomer in the membrane. However, how substrates reach the active site and how the characteristics of the active site tunnels determine the activity, specificity, and regioselectivity of PlaF for natural GPL substrates has remained elusive. Here, we combined unbiased and biased all-atom molecular dynamics (MD) simulations and configurational free energy computations to identify access pathways of GPL substrates to the catalytic center of PlaF. Our results map out a distinct tunnel through which substrates access the catalytic center. PlaF variants with bulky tryptophan residues in this tunnel revealed decreased catalysis rates due to tunnel blockage. The MD simulations suggest that GPLs preferably enter the active site with the $s n-1$ acyl chain first, which agrees with the experimentally demonstrated PLA 1 activity of PlaF. We propose that the acyl chain-length specificity of PlaF is determined by the structural features of the access tunnel, which results in favorable free energy of binding of medium-chain GPLs. The suggested egress route conveys fatty acid products to the dimerization interface and, thus, contributes to understanding the product feedback regulation of PlaF by fatty acid-triggered dimerization. These findings open up opportunities for developing potential PlaF inhibitors, which may act as antibiotics against $P$. aeruginosa. 


\section{Introduction}

Pseudomonas aeruginosa is an opportunistic and versatile pathogen, which causes infections in plants [1] and humans [2]. It is a multi-drug resistant Gram-negative bacterium and a frequent cause of nosocomial infections [3]. The pathogenicity of $P$. aeruginosa relies on both cell-associated and extracellular virulence factors [3]. Among those virulence factors are phospholipases $[4,5]$, including phospholipase $A_{1}\left(P L A_{1}\right)$, which hydrolyze cellular glycerophospholipids (GPLs) at the sn-1 position into lysoglycerophospholipids (LGPLs) and fatty acids (FAs) [6, 7].

GPLs primarily form bilayers, which maintain a permeability barrier for cells and organelles [8], while membrane-bound LGPLs can destabilize membrane integrity in Gram-negative bacteria $[9,10]$. GPLs [11] and LGPLs $[12,13]$ can regulate the function and stability of membrane proteins. Interestingly, biofilm formation and growth phase transitions in $P$. aeruginosa are accompanied by the alteration of membrane GPL composition [14, 15]. FAs belong to the diffusible signal factor family (DSF) and are possible signal molecules because they can diffuse through cell membranes and contribute to the regulation of diverse biological functions in various Gram-negative pathogens [16]. In P. aeruginosa, DSFs promote biofilm formation and antibiotic resistance $[17,18]$.

We recently identified $\mathrm{PlaF}$, an integral, inner membrane $\mathrm{PLA}_{1}$ that has a profound role for membrane GPL remodeling in $P$. aeruginosa. Furthermore, a $P$. aeruginosa $\Delta p l a F$ knockout strain showed strongly attenuated virulence in Galleria mellonella and human macrophages models compared to the wild-type, which suggests that PlaF-mediated GPL remodeling contributes to the virulence of $P$. aeruginosa [19]. Crosslinking (in vivo and in vitro) and micro-scale thermophoresis experiments showed that PlaF exists in both monomeric and dimeric configurations, although it is active only in the monomeric state [19]. The crystal structure of PlaF revealed that a homodimer is formed by interactions between the transmembrane (TM) and juxtamembrane (JM) regions [19]. The homodimer contains co-crystallized endogenous ligands, myristic acid (MYR) and 
undecanoic acid (UND) from $P$. aeruginosa, (Figure 1), which are non-covalently bound in the active site cavity [19]. Moreover, a complex T-shaped active site pocket formed by the TM, JM, and catalytic domain revealed three openings, one at the dimer interface, one close to the catalytic serine (S137), and one most likely pointing towards the membrane [19]. Molecular simulations of PlaF activation revealed a mechanism that involves a dimer-to-monomer transition followed by tilting of the monomer in the membrane [19]. The tilting orients PlaF in a configuration relative to the membrane such that substrates can directly access the cleft (Figure 1). By contrast, in the configuration observed in the crystal structure, the residues lining the opening of the active site cleft are more than $5 \AA$ above the membrane surface (Figure 1). However, it is unknown how substrates reach the active site and how the characteristics of the active site determine the activity, specificity, and regioselectivity of PlaF for mediumchain GPLs.

A

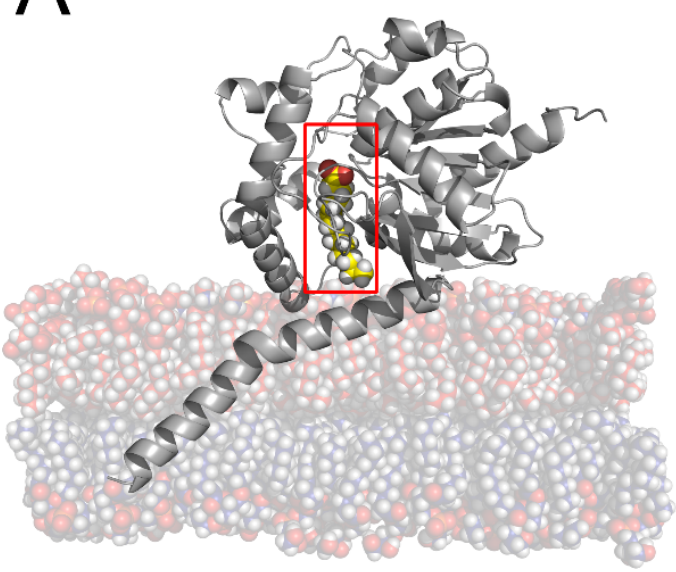

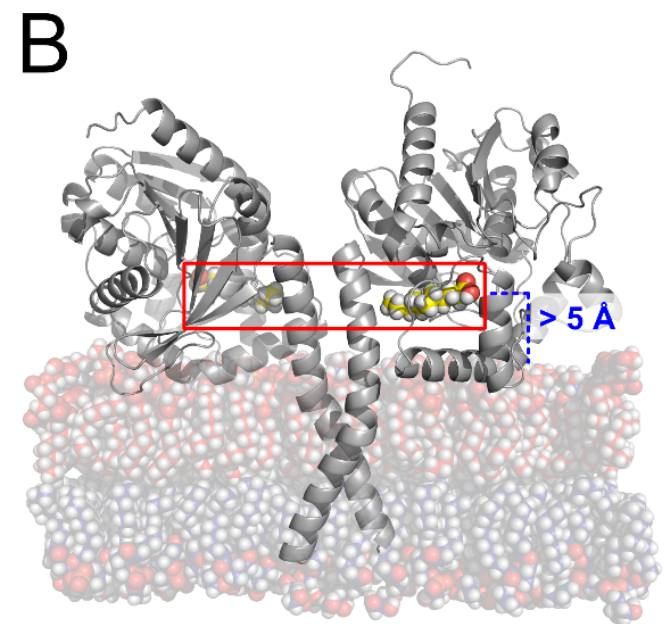

Figure 1: Schematic representation of the orientation of PlaF in the membrane. A) Chain A of dimeric $\mathrm{PlaF}$ in the tilted state; this state allows direct contact of the active site tunnel (red box) with the membrane. Yellow spheres represent the $\mathrm{C}$ atoms of the co-crystallized PlaF product, MYR. B) In dimeric PlaF, the active site tunnel is located $>5 \AA$ above the membrane. Yellow spheres represent the $C$ atoms of the co-crystallized PlaF products, MYR (left) and UND (right), within the active site tunnel.

The molecular mechanism underlying the access and binding of GPL to PLA is poorly understood in general because only a few PLA structures from microorganisms have been resolved, which either revealed closed conformations of their phospholipase domains $[4,20]$ or an accessible pocket that is predominantly hydrophobic [21] or 
amphipathic [22]. For the latter, regioselectivity was suggested to be achieved through binding of the GPL phosphate group to the polar pocket, which constrains the sn-1 acyl chain in a neighboring hydrophobic pocket [22]. Finally, structural analysis of the outer membrane PLA (OMPLA) from Escherichia coli in the complex with an inhibitor provided information about GPL recognition by this PLA [23, 24]. However, OMPLA is an integral $\beta$-barrel protein with a hydrophobic GPL binding cleft and the active site located at the $\beta$-barrel exterior. Hence, the mechanism by which PlaF recognizes GPL substrates must be conceptually different from that of OMPLA.

Here, we combined unbiased and biased all-atom molecular dynamics (MD) simulations and configurational free energy computations to identify access pathways to the catalytic center of $\mathrm{PlaF}$. The results were validated by mutational and enzymatic studies on PlaF variants with blocked substrate access. Our results map out a distinct tunnel for substrate access within PlaF, provide explanations for the substrate specificity and PLA 1 activity of PlaF, and suggest egress routes for hydrolysis products. These findings enhance our understanding of the mechanism by which membrane protein function is regulated through protein-GPL interactions.

\section{Results}

\section{Access pathways to the catalytic site in PlaF}

The crystal structure of PlaF revealed three pronounced tunnels, forming a large, Tshaped active site cleft. This cleft is compatible with binding bulky GPL substrates [19]. However, the structural dynamics of biomolecules may lead to variations in the tunnel shape [25]. Therefore, we reanalyzed trajectories from 10 replicas of unbiased MD simulations of $2 \mu$ s length for each of the systems di-PlaF (dimeric PlaF), $\mathrm{PlaF}_{\mathrm{A}}$ (chain A from the crystal structure), $\mathrm{PlaF}_{\mathrm{B}}$ (chain $\mathrm{B}$ from the crystal structure), and $\mathrm{t}-\mathrm{PlaF}_{\mathrm{A}}$ (chain A from the crystal structure in a tilted orientation) from our previous work [19] using CAVER [26]. CAVER analyzes and visualizes tunnels and channels in protein structures. 


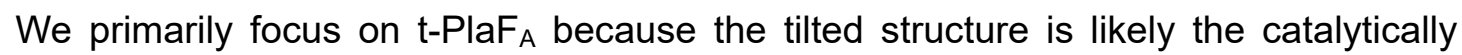
active form [19]. We identified the three tunnels that connect the active site of $t-\mathrm{PlaF}_{\mathrm{A}}$ to its surface like in the crystal structure (Figure 2) [19]: Tunnel 1 (T1) and tunnel 2 (T2) point towards the membrane, and tunnel 3 (T3) opens to the periplasmic space $>15$ $\AA$ above the membrane (Figure 2). T1 and T2 converge close to the active site and connect to T3. In the crystal structure, T1 contains MYR (chain A) and UND (chain B), which are hydrolysis products of GPL substrates with C14 and C11 acyl chain(s), respectively.
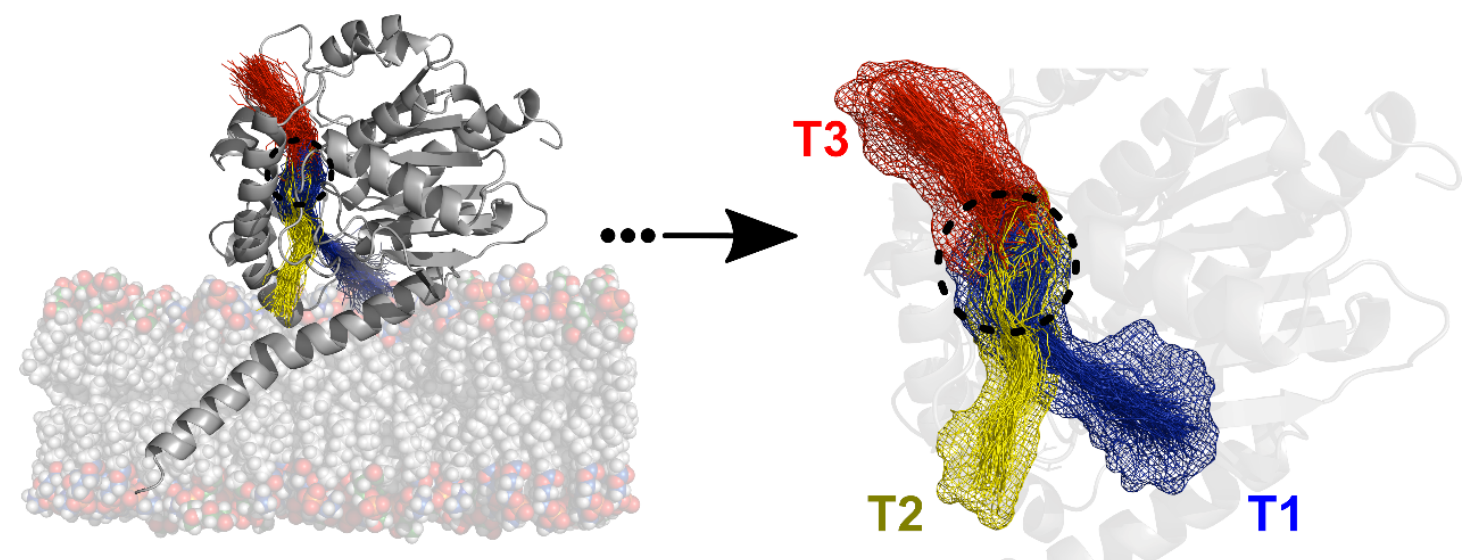

154

155

156

157

158

159

Figure 2: Clusters of tunnels identified in $\mathrm{t}-\mathrm{PlaF}_{\mathrm{A}}$ ensembles. Three major tunnel clusters connect the catalytic site (black dashed circle) of PlaF to the protein surface. Tunnels $\mathrm{T} 1$ and T2 point towards the membrane; tunnel T3 is located $>15 \AA$ above the membrane, with its opening pointing into the periplasmic space.

Table 1: Characteristics of tunnel clusters identified from unbiased MD simulations of $t-\mathrm{PlaF}_{\mathrm{A}}$ using CAVER.

\begin{tabular}{|c|c|c|c|c|}
\hline $\begin{array}{c}\text { Tunnel } \\
\text { cluster }\end{array}$ & Occurrence $^{\text {a,b }}$ & $\begin{array}{c}\text { Maximum } \\
\text { bottleneck radius }^{\text {c }}\end{array}$ & $\begin{array}{c}\text { Average } \\
\text { bottleneck radius }^{c}\end{array}$ & $\begin{array}{c}\text { Average } \\
\text { length }^{\mathbf{c}}\end{array}$ \\
\hline T1 & 30.45 & 3.18 & 2.28 & 27.08 \\
\hline T2 & 21.80 & 2.95 & 2.21 & 23.75 \\
\hline T3 & 27.75 & 3.13 & 2.29 & 15.16 \\
\hline
\end{tabular}

a Snapshots in which the tunnel is identified with respect to the total number of snapshots, in \%.

${ }^{\mathrm{b}}$ Data calculated with a probe radius of $2.0 \AA$.

c In Å.

T1 is the longest tunnel (Table 1) and was open more often than the other two tunnels 
167 (Table 1). The tunnel radii fluctuate between $2 \AA$ and $5 \AA$ depending on the location in 168 the tunnel and the simulation length (Figure S1). The average bottleneck (narrowest 169 part of the tunnel) radius of all tunnels is $2.26 \pm 0.02 \AA$ (mean \pm standard error of the 170 mean), which is close to the radius of glycerol (2.74 $\AA$ ) [27], an essential component 171 of all GPLs, but smaller than the radius of 1,2-dilauroyl-sn-glycero-3-phosphoglycerol 172 (DLPG) ( 4.4 $\AA$ ) deduced from the lipid's area-per-lipid [28]. For comparison, tunnels 173 in monomeric $\mathrm{PlaF}_{\mathrm{A}}, \mathrm{PlaF}_{\mathrm{B}}$, and the two chains of di-PlaF show open occurrences of $174 \sim 20 \%$ to $\sim 5 \%$ (Table S1), indicating no marked differences between monomeric and di-PlaF.

To conclude, the active site of PlaF is connected to its surface with three tunnels. In the $\mathrm{t}_{-\mathrm{PlaF}}$ configuration, only $\mathrm{T} 1$ and $\mathrm{T} 2$ allow direct access of GPL or LGPL substrates from the membrane.

\section{PlaF preferentially hydrolyses medium-acyl chain LGPLs}

Previously, we showed that PlaF can produce LGPLs by releasing FAs bound to the sn-1 position of GPLs. Here we experimentally tested if purified PlaF in vitro hydrolyses LGPLs by quantifying fatty acids released from a range of LGPLs varying in the head group and acyl chain length $(\mathrm{C} 14-\mathrm{C} 18)$. The results revealed that PlaF can hydrolyze all tested LGPLs, with a preference for medium-acyl chain LGPLs (Figure 3). Interestingly, the lysoPLA activity of PlaF was 10- to 100-fold higher for all LGPLs than

187 its PLA activity [19], indicating that hydrolysis of the first acyl chain in GPLs is much slower than that of the second one. 


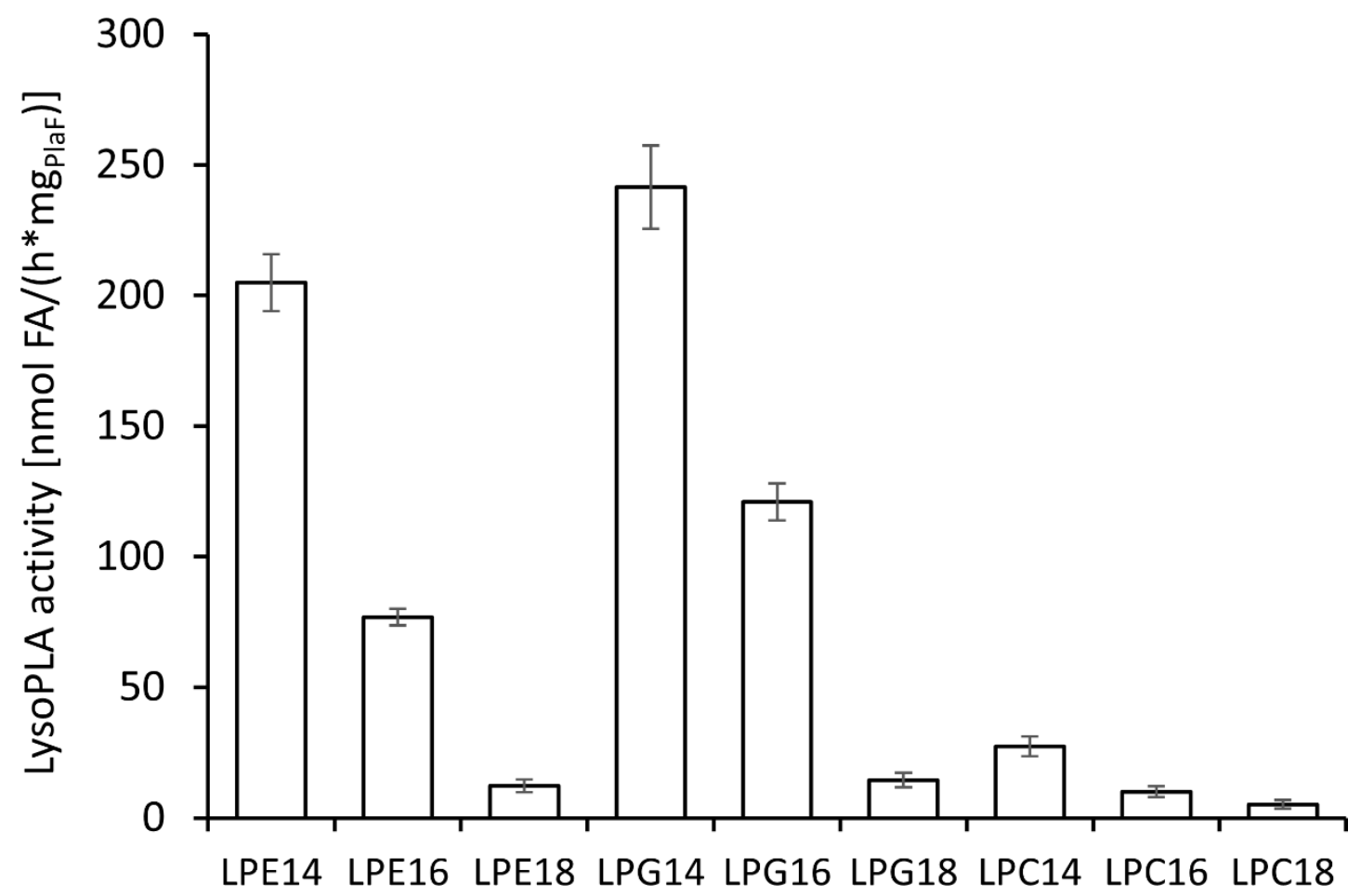

Figure 3: Lysophospholipase A activity of PlaF. Assays were performed by incubating purified PlaF ( $8 \mathrm{nmol})$ in $n$-dodecyl $\beta$-D-maltoside (DDM) micelles with the substrate, followed by quantification of released FA by NEFA-assay. Lyso-phosphatidylethanolamine (LPE), lysophosphatidylglycerol (LPG), and lyso-phosphatidylcholine (LPC) contain fatty acids with 14 - 18 carbon atoms. The results are the means \pm standard deviation of three independent experiments.

\section{GPL and LGPL substrate extraction into solvent and acyl chain mobility}

For probing the energetics of GPL and LGPL substrate extraction from the membrane into the solvent, we computed the free energy profile for DLPG and 1-myristoyl-2hydroxy-sn-glycero-3-phosphoglycerol (2LMG) extraction (Supplementary results), which resulted in free energy differences between the two states of $\sim 13 \pm 0.1 \mathrm{kcal} \mathrm{mol}^{-1}$ and $\sim 8 \pm 0.3 \mathrm{kcal} \mathrm{mol}^{-1}$ (Figure S2A), in very good agreement with the excess chemical potential related to these lipids' critical micelle concentration (CMC). For access to T3, substrates would need to leave the membrane and pass through the water phase, which makes this route energetically unfavorable. Hence, T3 was not considered for further analyses. 
As T1 and T2 are immersed in the hydrophilic membrane surface (Figure S3A), access of GPL and LGPL substrates to the tunnels via the head groups is plausible. However, the tunnels' diameters are much smaller than that of a GPL like DLPG while in the membrane (see above). To explore the possibility that lipids access via their acyl chain instead, we probed how frequently the terminus of a GPL's acyl chain can reach the membrane interface. The probability distribution of GPL's acyl chains with respect to the coordinate perpendicular to the membrane (z-coordinate) was determined during the last 40 ns of 300 or 100 ns long MD simulations for membrane bilayers with or without $\mathrm{t}-\mathrm{PlaF}_{\mathrm{A}}$, respectively (Figure S3A). Tails from both the upper and lower leaflet were considered. Positive $z$-coordinate values indicate that a tail moves towards the water-membrane interface of its leaflet; negative values indicate that it moves towards the interface of the opposite leaflet. The peak of the probability distributions is at $z \approx 2 \AA$ indicating the mobility of lipid termini within the leaflet (Figure S3A, see also Movie S1 and Movie S2). The interface of the simulated membrane is at $z \approx 10 \AA$ (Figure S3B). Notably, the cumulative probability of finding an acyl chain terminus at $z>10 \AA$ is $1.5 \%$ and $1.0 \%$ for systems with or without PlaF, respectively. Hence, there is a finite likelihood that acyl chain termini can reach the entrances of T1 and T2. This result is also supported by the electron density profiles of the membrane components (Figure S3B).

To conclude, for t-PlaF, the access route of substrates to T3 is energetically unfavorable. By contrast, acyl chain termini of GPL lipids can reach the entrances of T1 and T2 during the time scales of our MD simulations.

\section{Access modes of GPL and LGPL substrates into PlaF}

As a prerequisite to computing the energetics of substrate access to the active site of PlaF, we aimed to identify favorable access modes. We applied steered molecular dynamics (sMD) simulations [29] to pull substrates inside T1 and T2 (Figure 4) via head access first or tail access first. 
The closest substrate to the tunnel entrance was chosen for sMD simulations. The terminal oxygen and nitrogen atom of phosphatidylglycerol (PG) or phosphatidylethanolamine (PE) head groups, respectively, were considered for head access pulling. For tail access, the terminal carbons of respective acyl chains were considered. Substrates from the membrane were initially pulled through consecutive virtual points in T1 or T2 using four or five steps, respectively (Figure S4A, Table S2). However, pulling with terminal atoms leaves the cleavage site of the substrate distant to the catalytic S137 (Figure S4B). Therefore, the substrates were further pulled into T3, using three additional steps (Figure S4A). Depending on the access mode, the sn1 or sn-2 sites of respective substrates were further pulled towards the nucleophilic $\mathrm{OH}$ group of the catalytic S137 (Table S2). Finally, this resulted in pulling pathways subdivided into eight and nine steps for T1 and T2, respectively (Table S2).

As a reaction coordinate, the distance between the pulled atom of a substrate and the consecutive virtual point was used. For each step, we repeated the pulling 50 times and computed the work done as a function of the reaction coordinate. By applying Jarzynski's relation (eq. 1) [30], the work was related to the free energy difference between the two states of the pulling simulation. The sMD trajectory whose workversus-reaction coordinate profile is closest to the Jarzynski average (eq. 1) was considered most favorable. Its endpoint provided the starting point for the SMD simulations in the next part of the pulling pathway. As a result, the access pathway is close to the lowest-free energy pathway of substrate access to the catalytic site. Overall, this approach is the reversed version of sampling unbinding trajectories of ligands from proteins before applying Jarzynski's relation [31-33] but uses piecewise sMD simulations along the pathway to account for the curvilinear tunnels. A total of $\sim 27$ $\mu$ s of sMD simulation time was used for either tunnel (Table S3).

The activity of PlaF for GPL decreases with the increasing lengths of the acyl chain between $\mathrm{C} 12$ and $\mathrm{C} 18$, irrespective of the type of head group, PG or PE [19]. In addition, the number of acyl chains in a substrate also influences the PlaF activity, with 
which PlaF is most active [19], 1,2-dilauroyl-sn-glycero-3-phosphorylethanolamine (DLPE), 1,2-distearoyl-sn-glycero-3-phosphoglycerol (DSPG), and 2LMG, a LGPL, for generating access modes (Figure 4A). Figure 4 exemplarily shows illustrations of the three access types for DLPG (see also Movies S3 - S8). Work-versus-reaction coordinate profiles for all pulling simulations related to DLPG access are shown in Figure S5 for T1 and Figure S6 for T2. Based on computed potentials of mean force (PMF) to evaluate the energetics of the access modes (see the next chapter), only tail access was considered for sMD simulations of the other GPL substrates (Figure S7). For 2LMG, head and tail access were considered for SMD simulations. generated for T1 and T2, resulting in 18 access modes in total.

A
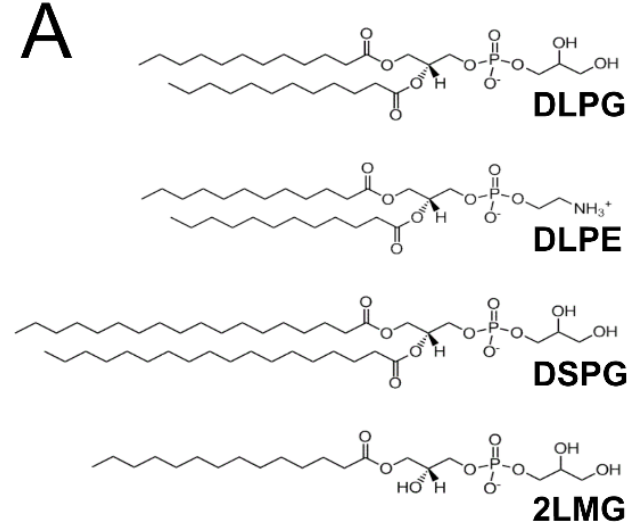

2LMG

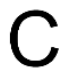

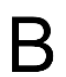

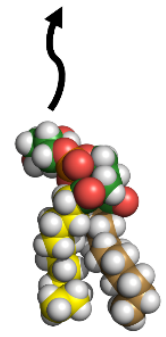

(i)

$D$

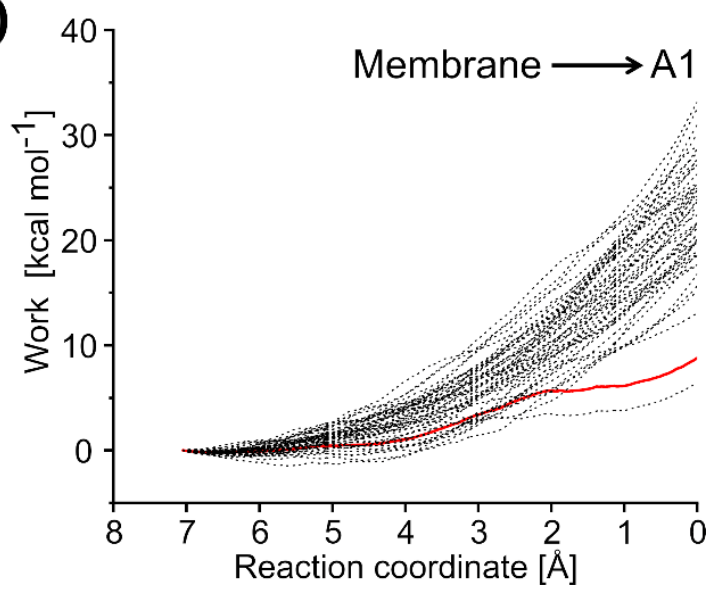

Figure 4: Illustration of the substrate access in $t_{-P l a F_{A}}$ A) Investigated GPL substrates, 


\section{1,2-dilauroyl-sn-glycero-3-phosphoglycerol}

(DLPG)

1,2-dilauroyl-sn-glycero-3phosphorylethanolamine (DLPE), 1,2-distearoyl-sn-glycero-3-phosphoglycerol (DSPG), and LGPL substrate, 1-myristoyl-2-hydroxy-sn-glycero-3-phosphoglycerol (2LMG). B) Possible modes by which a GPL can access a tunnel (indicated with black arrow): with its head (green spheres represent the $C$ atoms) first (i), tail 1 (yellow spheres represent the $C$ atoms) first (ii), or tail 2 (orange spheres represent the $\mathrm{C}$ atoms) first (iii). C) PlaF is embedded in a membrane consisting of DLPE (head group $C$ atoms as blue spheres) and DLPG (head group $C$ atoms as green spheres) at a ratio of 3:1. The DLPG closest to the entrance of T1 (acyl chains colored) is shown while being loaded by its head, in the direction indicated with a blue arrow. A segmented path was considered for substrate access. T1 was segmented into four parts, and T3 into three parts, which are used as pulling points in SMD simulations. Depending on the access mode, in the last pulling step, the $s n-1$ or $s n-2$ site of the substrate is further pulled towards the nucleophilic $\mathrm{OH}$ group of the catalytic S137, resulting in, in total, 8 steps. A similar approach was used for T2 (Figure S4A). D) For the first segment of T1 (i.e., A1), the work done (black lines) during 50 independent replicas of SMD simulations to pull the DLPG from the membrane is plotted against the reaction coordinate. The coordinates of the replica, the workversus-reaction coordinate profile of which is closest to the Jarzynski's average (red line), are considered for pulling in the next segment. See the SI for plots of all other SMD simulations.

\section{Potentials of mean force of DLPG access modes}

PMFs were computed from umbrella sampling (US) simulations [34] and postprocessing with WHAM $[35,36]$ to evaluate the energetics of substrate access for the access modes described in the previous chapter (Figure 4). As a reaction coordinate, the distance between the center of mass (COM) of the three oxygen atoms of the glycerol moiety in the substrate to the COM of $\mathrm{C}_{\alpha}$ atoms of the catalytic residues $\mathrm{S} 137$ and H286 was used. Residue D258 was not included in the reaction coordinate, as its side chain is distant from the active site (Figure S4A). As the tunnels are almost straight, the reaction coordinate monotonically decreases as the substrate approaches the active site from the membrane (Figure S4B). Initially, we focused on the US simulations for the best PlaF substrate [19], DLPG. PMFs were calculated for the three access modes of DLPG across either tunnel, T1 and T2. The PMFs were evaluated for convergence, excluding the first 200 ns of 300 ns sampling time. PMFs were found converged by $300 \mathrm{~ns}$, yielding a maximal difference of $\sim 1 \mathrm{kcal} \mathrm{mol}^{-1}$ as to a PMF computed from 280 ns per window (Figure S8). The median overlap between the 
312 reaction coordinate distributions of neighboring windows was sufficient $(\geq 4.8 \%$ and

$313 \quad 3.5 \%$ for T1 and T2, respectively) (Figure S9).

314 The PMFs of DLPG access modes show marked differences (Figure 5A). Access with

315 the head first is the least favorable for both T1 and T2, resulting in steep PMFs with

316 free energy barriers of 11 and $9 \mathrm{kcal} \mathrm{mol}^{-1}$ (Figure 5A), in contrast to tail access. Most

317 of the residues within a radius of $3 \AA$ in T1 and T2 have either a neutral non-polar side

318 chain, which likely facilitates tail access to the active site of PlaF. Furthermore, access

319 with either one of the two tails first is more favorable in T2 than T1 (Figure 5A). Finally,

320 access with tail 1 first in T2 is most favorable and results in no free energy barrier until

321 the substrate reaches the active site (Figure 5A). As the two acyl chains of DLPG are

322 identical, these results suggest that their connection with the glycerol moiety causes

323 differences in how the lipid interacts with the tunnel, which may explain how PlaF

324 achieves regioselectivity to exert its $\mathrm{PLA}_{1}$ function. 
A
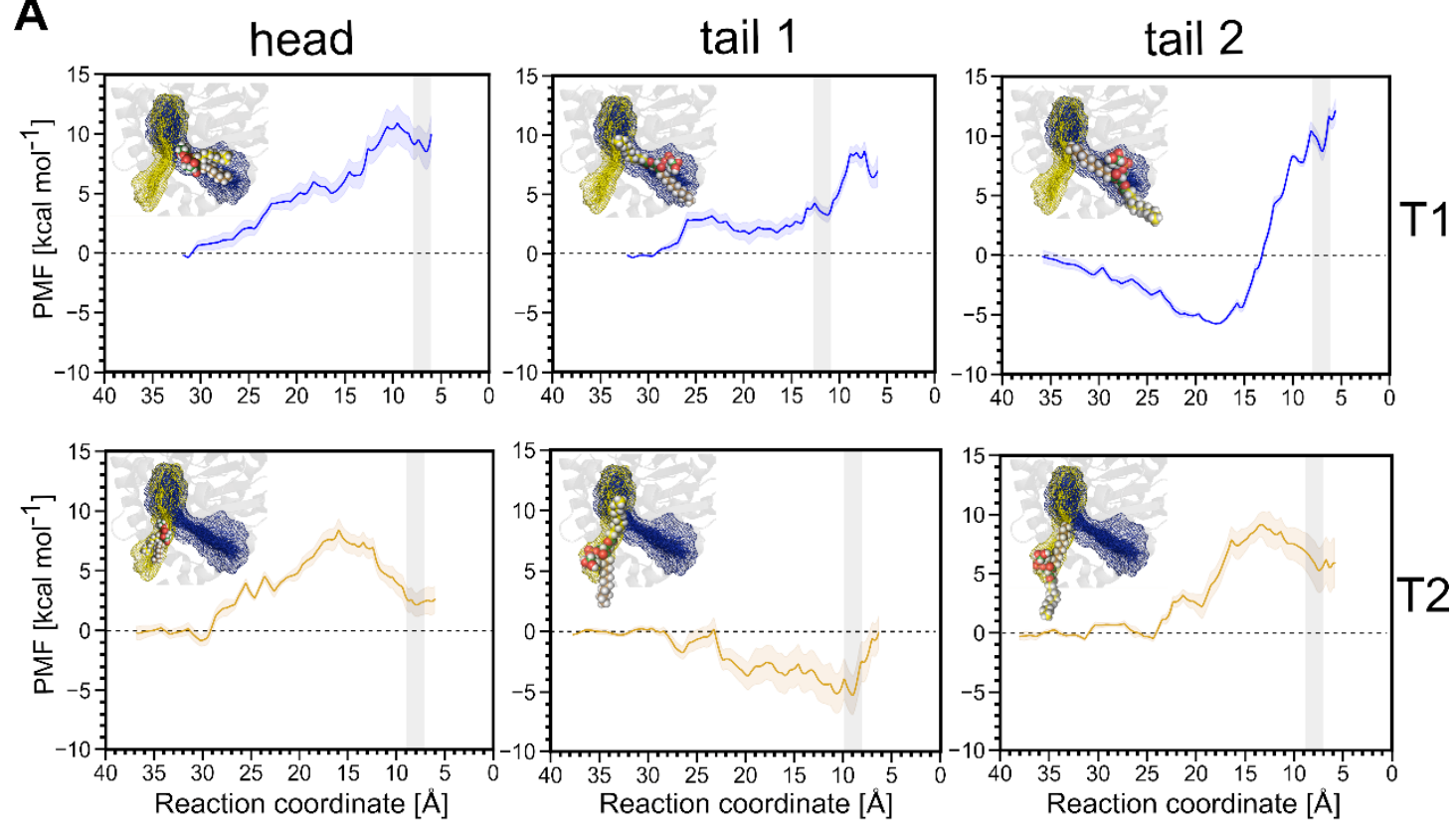

B
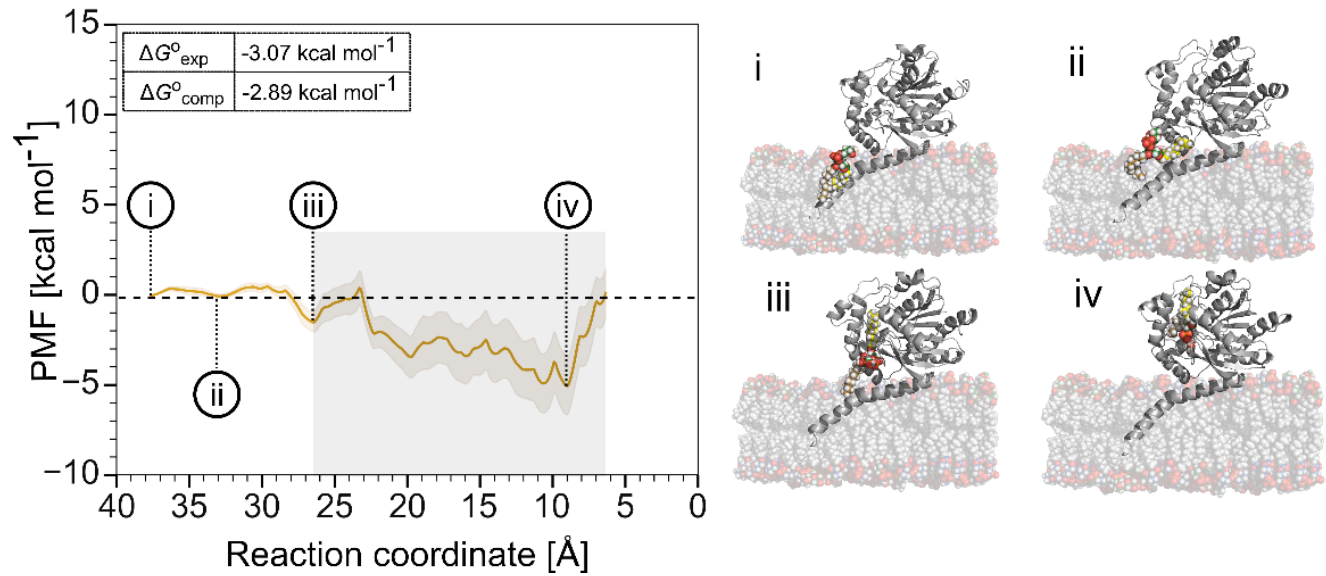

Figure 5: Potential of mean force profiles for DLPG access. (A) PMFs of three access modes (head, tail 1, tail 2; see Figure 4B) of DLPG in T1 (blue curve) and T2 (yellow curve). For both tunnels, access with tail 1 first yields the lowest free energy barriers to reach the active site. Furthermore, DLPG access into T2 with tail 1 first is overall the most favorable. The catalytic site is marked with a grey box. Insets within the plots illustrate the different DLPG access modes into the respective tunnels. (B) States during DLPG access via tail 1 through T2, shown on the right, are marked in the PMF profile (left). The grey box corresponds to the integration limits used to calculate $K_{\text {eq }}$ (eq. 2 ) to determine $\Delta G^{\circ}$ comp (see inset). State i: The starting position of DLPG (in the membrane). State ii: Tail 1 reaches the surface of the membrane close to the entrance of T2. State iii: Tail 1 enters inside T2, while tail 2 remains within the membrane. State iv: $s n-1$ site of tail 1 reaches the catalytic site.

To validate our results, we computed the absolute binding free energy of DLPG to PlaF from the PMF for tail 1 access in $\mathrm{T} 2, \Delta \mathrm{G}_{\text {comp }}^{\circ}=-2.89 \pm 1.46 \mathrm{kcal} \mathrm{mol}^{-1}$ (eq. 4).

Assuming that product formation is slower than substrate dissociation from an enzyme, 
340 the Michaelis constant $K_{\mathrm{m}}$ is equal to the dissociation constant $K_{\mathrm{D}}$ of the enzyme341 substrate complex $[37,38]$. Under this assumption, from $K_{m}=7.612 \pm 1.907 \mathrm{mM}$ for 342 DLPG in PlaF [39], the experimental binding free energy $\Delta \mathrm{G}_{\text {exp }}^{\circ}=-3.07 \pm 0.30 \mathrm{kcal}$ $\mathrm{mol}^{-1}$ at $T=303 \mathrm{~K}$ is calculated, which is within chemical accuracy [40] of $\Delta \mathrm{G}_{\text {comp }}^{\circ}$. We also computed $\Delta \mathrm{G}_{\text {comp }}^{\circ}$ for the other five access modes of DLPG (eq. 4). The lowest $\Delta \mathrm{G}_{\text {comp }}^{\circ}$ among all six modes was obtained for tail 2 access in T1 (Table S4). However, the PMF profile (Figure 5A) reveals that the configurational free energy minimum is not situated close to the active site but in the middle of T1. Here, one of the tails is still in the membrane, while the other is being loaded into the tunnel. If the PMF profile is integrated with two separate parts, first, a negative free energy for tail access into the tunnel results, followed by a positive free energy to reach the active site. This suggests that this access mode cannot yield a catalytically active configuration. For the other four access modes, $\Delta \mathrm{G}_{\text {comp }}^{\circ}>0.96 \mathrm{kcal} \mathrm{mol}^{-1}$ (Table S4). These findings corroborate tail 1 access of DLPG in T2 as the most likely access mode.

Along the PMF of tail 1 access of DLPG in T2, four distinct states can be identified (Figure 5B). The two tails of DLPG are immersed in the membrane at a reaction entrance of T2 (state ii). This is concordant with the tail distributions along the zcoordinate during unbiased MD simulations (Figure S3), indicating that tail termini can reach one of the access tunnels of $\mathrm{t}^{-} \mathrm{PlaF}_{\mathrm{A}}$ without a considerable energetic cost. Once tail 1 enters T2, the PMF becomes negative (state iii), indicating that DLPG access that way is favorable. Finally, at $\sim 8 \AA$ of the reaction coordinate, the PMF has a global minimum (state iv). There, tail 1 is located in T3, and the acyl moiety at the sn-1 position of DLPG is close to the catalytic S137 of PlaF (Figure S10B) such that a nucleophilic attack can commence. with tail 1 first is most favorable there. This is in line with PlaF being a PLA ${ }_{1}$, which 
cleaves its substrates at the sn-1 position. As of T3, it is likely essential for substrate access by allowing to accommodate the substrate tail to be hydrolyzed by PlaF.

\section{Potentials of mean force for accesses of other substrates}

Considering the results for DLPG, we performed US simulations for DSPG and DLPE only for tail 1 access. For the LGPL substrate, it has remained undetermined if the head or tail access is energetically favorable; hence, we performed US simulations for both access modes of 2LMG. As for DLPG, T2 is preferred over T1, regardless of the access modes (Figure 5A), we only considered T2 for computing PMFs for the other substrates. Similar to DLPG, the PMFs converged at 300 ns of sampling time, yielding a maximal difference of $\sim 0.5 \mathrm{kcal} \mathrm{mol}^{-1}$ as to a PMF computed from $280 \mathrm{~ns}$ per window (Figure S11). Neighboring umbrella windows have a sufficient median overlap $\geq 3.2 \%$ (Figure S12).

For DSPG and DLPE, access with tail 1 first in T2 results in pronounced free energy barriers of 11 and $14 \mathrm{kcal} \mathrm{mol}^{-1}$ (Figure 6A, B), in contrast to DLPG $\left(0.5 \mathrm{kcal} \mathrm{mol}^{-1}\right)$. This finding indicates that a longer acyl chain or a neutral head group makes substrate access to PlaF disfavorable, which coincides with lower PlaF activities for such substrates [19]. For 2LMG, access with the tail first is more favorable than with the head, as for DLPG (Figure 6C, D). Furthermore, tail access by 2LMG leads to a free energy barrier lower by $\sim 6.5 \mathrm{kcal} \mathrm{mol}^{-1}$ than those for tail access by DSPG and DLPE (Figure 6A, B, D), which is concordant with the activity profile of PlaF [19]. 
A

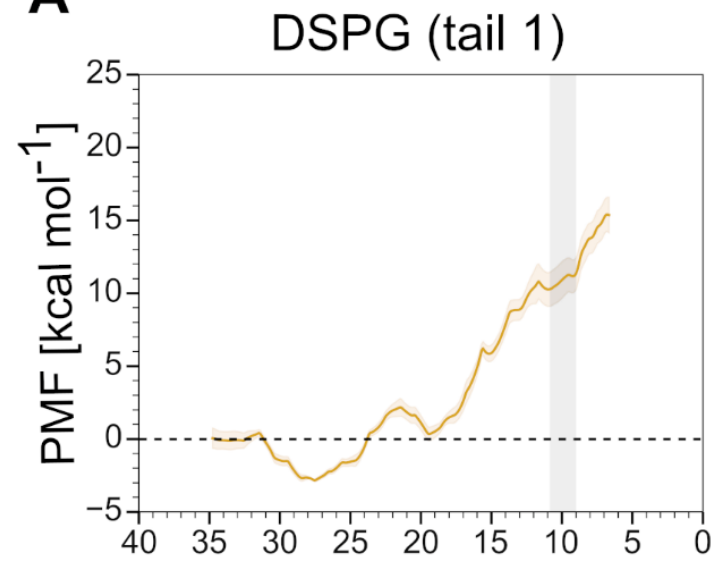

C

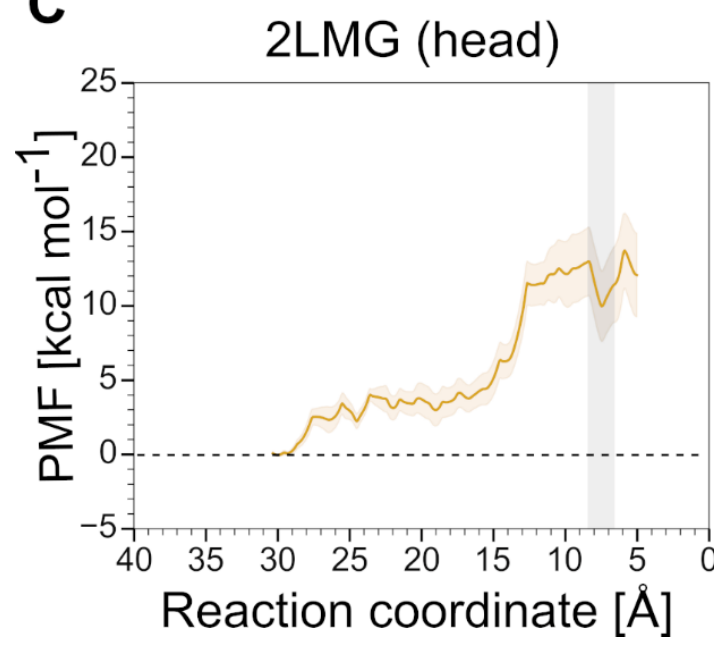

B

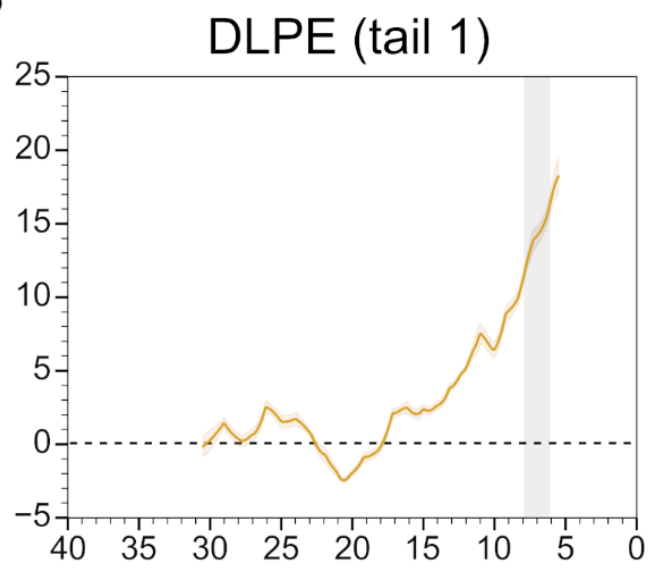

D

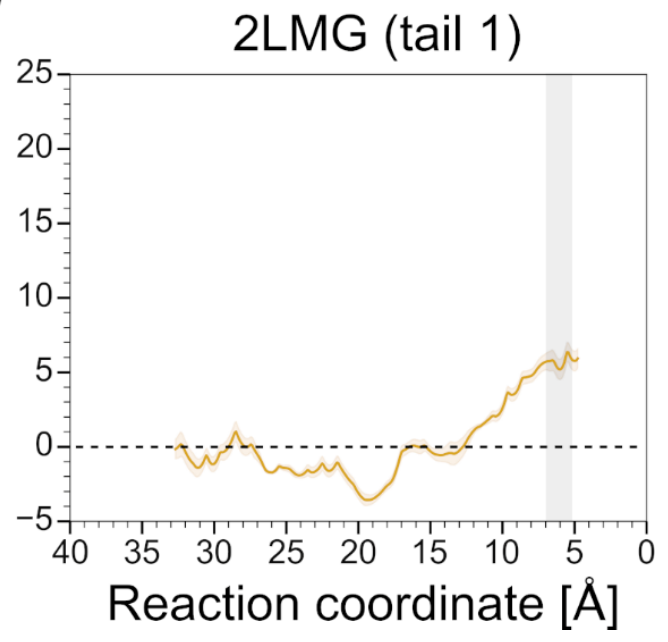

Figure 6: PMF profiles for other substrates across T2. Four systems were investigated to reveal the energetics of DSPG access via tail $1(A)$, DLPE via tail $1(B), 2 L M G$ via head $(C)$, and 2 LMG via tail 1 (D). Among these substrates, access of $2 L M G$ via tail 1 has the lowest free energy barrier. The catalytic site is marked with a grey box.

To conclude, tail 1 access in T2 of GPL substrates with longer acyl chains or neutral head groups is disfavorable compared to DLPG access, in line with PlaF's substrate specificity. For the LGPL substrate 2LMG, tail 1 access is also favored over head access and more favorable than DSPG and DLPE access.

\section{Tryptophan substitutions in T2 hamper DLPG access}

To validate the prediction that $\mathrm{T} 2$ is the preferred access pathway, we identified residue positions in all identified tunnels that, when substituted with tryptophan (Trp), should constrict the tunnel and, thus, block substrate access. Earlier, this strategy has been 
used to block tunnels of a dehalogenase and influence its activity by limiting the rate of product release [41]. In the case of PlaF, the products are less bulky than the substrates, such that product release should be less impacted than substrate access due to constricted tunnels.

$\mathrm{PlaF}$ variants were predicted subject to minimizing the structural destabilization due to the Trp substitution and preferring sites within the tunnels that influence its geometric characteristics (Table S5). We predicted four Trp substitutions for T1 and five for T2, and T3 each (Table S5). With any one of these substitutions in place, the impacted tunnel could not be identified anymore by CAVER applying the previously used probe radius of $2 \AA$, but with a smaller probe radius of $1.2 \AA$ (Figure 7). This indicates their constriction, also displayed by the time evolution of the tunnel profiles of the PlaF variants compared to PlaF wild type (Figure S13).

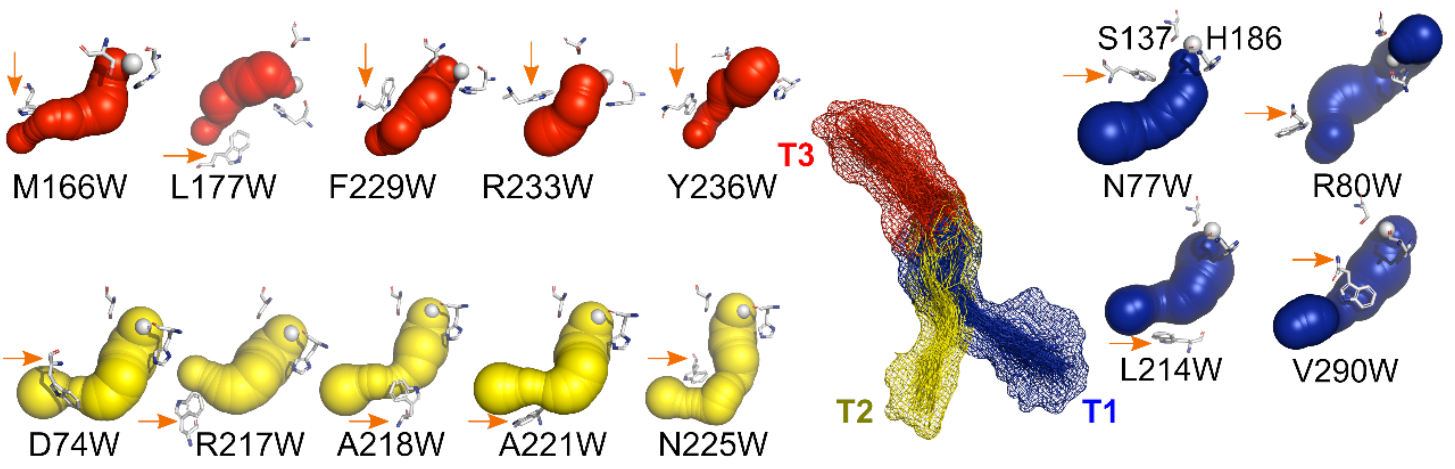

Figure 7: Influence of tryptophan substitutions on the radius of PlaF tunnels. The tunnels T1 (blue), T2 (yellow), and T3 (red) are identified by CAVER with a reduced probe radius of $1.2 \AA$, instead of $2 \AA$ used otherwise (Figure 2), showing that the tryptophan substitutions (orange arrows) narrow the tunnels. White spheres, wherever visible, represent the origin of the search defined by the COM of the catalytic residues $\mathrm{S} 137$ and $\mathrm{H} 286$.

The mutations of fourteen suggested residues (Figure 7) to Trp were generated by sequence- and ligation-independent cloning (SLIC) method in which the whole $p$-PlaF expression vector was amplified. Mutations were verified by sequencing, and the wild type PlaF $\left(\mathrm{PlaF}_{\mathrm{WT}}\right)$ and respective variants were produced in the homologous host, $P$. aeruginosa, following their immobilized metal affinity chromatographic (IMAC) purification from membranes solubilized with $n$-dodecyl $\beta$-D-maltoside (DDM) (Figure S14). All variants showed purity comparable to that of PlaFWT (Figure S14). The 
specific activity of PlaF variants and PlaFWT was analyzed by measuring the hydrolysis

of small ( $p$-nitrophenyl butyrate, $p$-NPB) and large (DLPG) substrates (Figure $8 \mathrm{~A}$ ). The activities of all nine variants in T1 and T3 measured with $p$-NPB and DLPG were similar lower activity with $p-N P B$ and DLPG than PlaFwt.

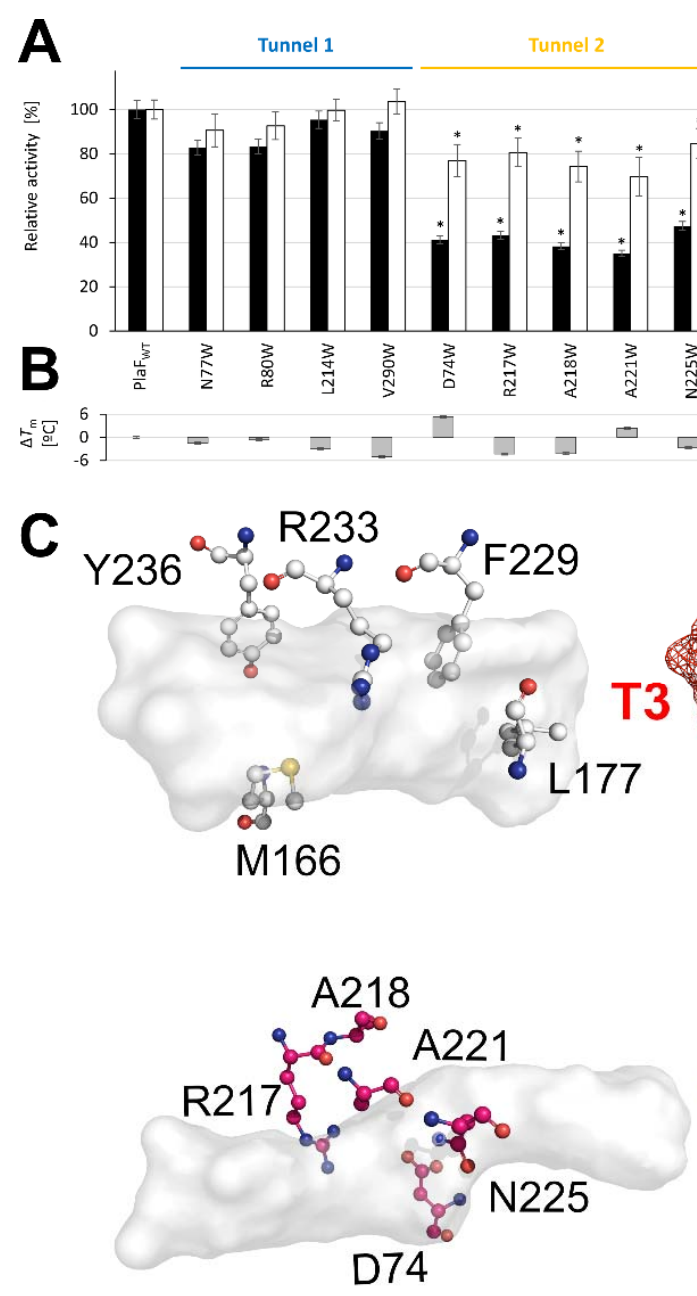

Figure 8: Lipolytic activity of PlaF and variants with Trp substitutions in T1-T3. A) Enzyme activities of purified PlaFWT and variants carrying respective substitutions measured with DLPG and $p$-NPB. Activities are normalized to the activity of PlaFWT, which was set as $100 \%$. Results are means \pm standard deviation of three independent measurements. Statistical analysis was performed using the t-test ( $\left.{ }^{*} p<0.001\right)$ of normally distributed values for DLPG $(n=8)$ and $p$ NPB $(n=9)$ measurements. B) The thermal stabilities of purified PlaFWt and variants were measured by nanoDSF. Results are shown as a difference in the melting temperatures $\left(\Delta T_{\mathrm{m}}\right)$ of the respective PlaF variant and PlaF ${ }_{W T}$, which was $57.3 \pm 0.2{ }^{\circ} \mathrm{C}$. Results are means \pm standard deviation of three independent measurements, each performed with three samples. C) The tunnels, T1 - T3 (mesh view in the center) are represented as white surfaces. The 
investigated amino acids are shown in ball-and-stick representation. The CPK coloring scheme was used to color all atoms except carbons, which vary from pink to white (see color scale) related to the PlaF activity for DLPG after substituting the corresponding residue for a tryptophan. The PlaF activity is reduced the most if Trp substitutions involve T2. D) Kinetic parameters of $\mathrm{PlaF}_{\mathrm{WT}}$ and the substrate binding-T2 variants $\mathrm{PlaF}_{\mathrm{D} 74 \mathrm{~W}}$ and $\mathrm{PlaF}_{\mathrm{A} 221 \mathrm{~W}}$ measured using $p$-NPB. Kinetic parameters were determined by non-linear regression analysis of the data ( $n=9$ for PlaFwt and PlaFA221w, $n=6$ for PlaF ${ }_{\mathrm{D} 74 \mathrm{w}}$ ) fitted to the Michaelis-Menten equation. The box plots represent the interquartile range between the first and third quartiles of the kinetic parameters determined for $\mathrm{PlaF}_{\mathrm{WT}}, \mathrm{PlaF}_{\mathrm{D} 74 \mathrm{~W}}$, and $\mathrm{PlaF}_{\mathrm{A} 221 \mathrm{~W}}$. The line inside the box is the median, and the whiskers represent the lowest and highest values. Statistical analysis was performed using the t-test $\left.{ }^{* * *} p<10^{-5}\right)$.

To exclude that a substitution leads to an unstable protein, we measured the thermal stability of each variant by detecting intrinsic protein fluorescence upon unfolding. None of the variants showed a drastically reduced stability (Figure 8B). On the other hand, two T2 variants were slightly more stable $\left(1.8-2.4^{\circ} \mathrm{C}\right)$ than the $\mathrm{PlaF} \mathrm{WT}_{\mathrm{T}}$, and three variants were slightly less stable $\left(2.7-4.3^{\circ} \mathrm{C}\right)$. Hence, Trp mutations do not affect PlaF's stability at the temperature of enzymatic assays $\left(30^{\circ} \mathrm{C}\right)$.

The observation that PlaF activities with DLPG and $p$-NPB predominantly decreased with substitutions in T2 (Figure 8A) indicates that substitutions with the bulky Trp impact passage through T2 (Figure $8 \mathrm{C}$ ). As expected, the activities with the larger DLPG decreased more $(52-65 \%)$ than with the smaller $p$-NPB $(15-30 \%)$.

We also determined kinetic parameters for the $p$-NPB hydrolysis of $\mathrm{PlaF}_{\mathrm{A} 221 \mathrm{~W}}$ and

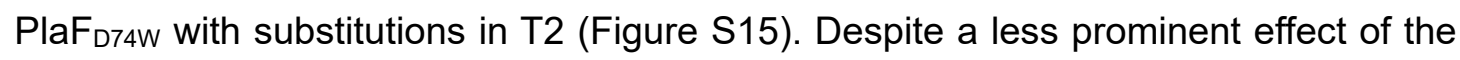
substitutions on specific activities measured with $p$-NPB than DLPG, $p$-NPB allows for reliable determination of PlaF activities over the range of substrate concentration and, thus, is applicable for the determination of kinetic parameters of PlaF. In contrast, enzyme kinetic experiments using hydrophobic DLPG are not feasible because of micelle formation and the slow rate of reaction.

Although, the affinities of $\mathrm{PlaF}_{\mathrm{WT}}, \mathrm{PlaF}_{\mathrm{A} 221 \mathrm{~W}}$, and $\mathrm{PlaF}_{\mathrm{D} 74 \mathrm{~W}}$ for $p$-NPB are similar (Figure $\mathrm{S} 15$, also see the table in the inset), the catalytic turnover of both variants $\left(\mathrm{PlaF}_{\mathrm{A} 221 \mathrm{~W}}\right.$ : $\left.k_{\text {cat }}=314.6 \pm 7.0 \mathrm{~s}^{-1} ; \mathrm{PlaF}_{\mathrm{D} 74 \mathrm{~W}:} k_{\mathrm{cat}}=403.4 \pm 15.1 \mathrm{~s}^{-1}\right)$ was significantly $\left(p<10^{-5}\right)$ lower 
474 than of PlaFWT $\left(k_{\mathrm{cat}}=487.8 \pm 15.4 \mathrm{~s}^{-1}\right)($ Figure 8D). These results confirm that the point

475 mutations PlaF $_{\mathrm{A} 221 \mathrm{~W}}$ and $\mathrm{PlaF}_{\mathrm{D} 74 \mathrm{~W}}$ interfere with $p$-NPB access through T2.

476 To conclude, biochemical studies of fourteen PlaF variants with Trp substitutions

477 introduced in all three tunnels showed tha only substitutions in T2 reduced lipolytic

478 activity of PlaF. These results confirm that $\mathrm{T} 2$ is the main route for substrate access

479 from the membrane to the catalytic site.

480

\section{Potential egress pathways of PlaF products}

482 Next, we aimed at identifying potential egress pathways for products of PlaF-catalyzed

483 hydrolysis. We performed a set of unbiased MD simulations starting from a hydrolyzed 484 2LMG in t-PlaF $\mathrm{A}$. The starting coordinates were taken from the last snapshot of the US 485 simulations of 2 LMG with tail 1 access through T2, considering the umbrella window 486 where the sn-1 position of 2LMG was closest to the catalytic site. Then, 2LMG was 487 cleaved into the respective products without changing their orientation in the tunnels 488 (Figure 9A). This led to MYR being in T3 at the beginning of the simulations and the 489 PGR (phosphatidylglycerol from LGPL, 2LMG) moiety pointing towards T2 (Figure 9B). 
A

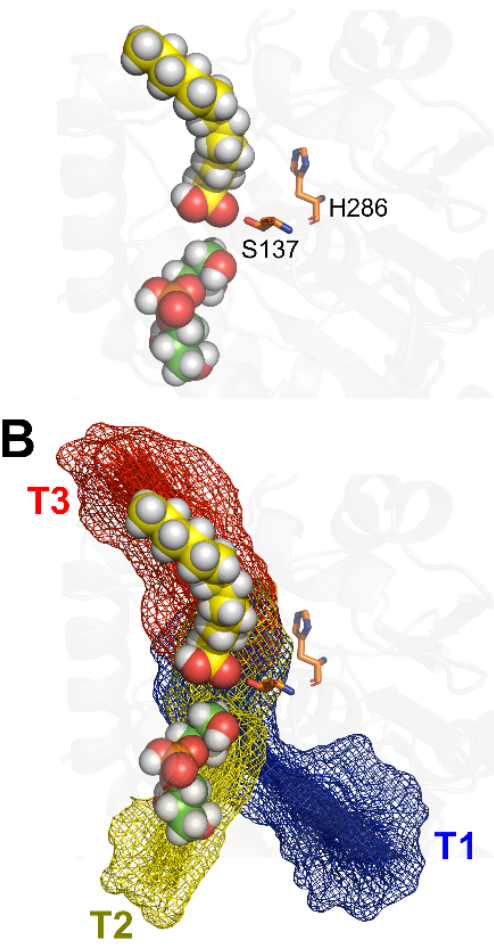

C
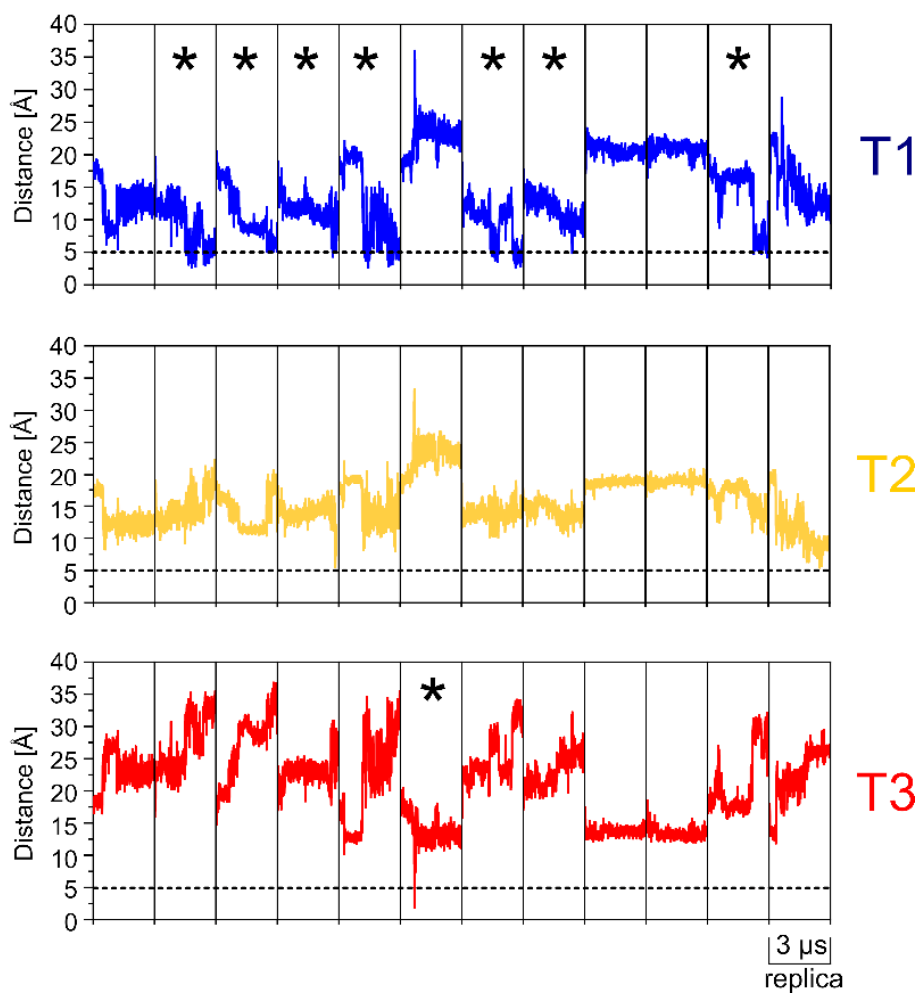

Figure 9: Unbiased MD simulations of $\mathrm{t}^{\mathrm{PlaF}} \mathrm{A}$ with bound hydrolysis products. (A) Starting configuration of the $2 \mathrm{LMG}$ products in $\mathrm{t}_{-\mathrm{PlaF}}$. MYR is represented with yellow spheres and PGR with green spheres. The catalytic S137 and H286 are shown as orange sticks. (B) The products are mapped over the respective tunnels. (C) The distance of MYR to the entrances of T1-T3 during 12 replicas of unbiased MD simulations of $3 \mu \mathrm{s}$. The dashed black line depicts the chosen cutoff of $5 \AA$, with replicas that reach this cutoff marked with an asterisk. MYR reaches a distance $\leq 5 \AA$ to the entrance of T1 in 7 replicas, in 1 replica for T3, and in none for T2. Note that in chain A of the PlaF crystal structure, MYR is found in T1.

In 12 replicas of $3 \mu$ s length each, the products relocated within the tunnels, sometimes even diffusing into the solvent (PGR moiety in 2/12 replicas via T1 and 3/12 replicas via T2; Figure S16). MYR relocated from its original position in T3 and approached the other tunnels of PlaF during the course of the MD simulations (Figure 9C). To deduce the displacement of MYR, we measured the distance of the carboxyl carbon to the entrance of each tunnel. A cutoff of $5 \AA$, according to previous studies [42-44], was used to identify those replicas where MYR reaches close to the tunnel entrance. MYR moved in $7 / 12$ replicas to the entrance of $\mathrm{T} 1$ and in $1 / 12$ replicas to the entrance of $\mathrm{T} 3$; the entrance of T2 was not reached (Figure 9C). Interestingly, the instance of MYR reaching the entrance of T3 flips within T3 such that the carboxyl group points to the 
510 entrance, rather than to the active site as after hydrolysis (Figure 9B). Altogether, MYR

511 reaches the entrance of T1 significantly more frequently than T2 $(p=0.0008)$ and T3

$512(p=0.0047)$ (Figure S17, Table S6).

513 To conclude, hydrolysis products of 2LMG diffuse within PlaF during time scales of

$5143 \mu \mathrm{s}$, sometimes also between tunnels. T1 and, to a lower extent, T3 are the most likely 515 egress pathways of FAs from PlaF, although more sampling is required to observe 516 actual egress.

\section{Discussion}

Dimer-monomer transitions regulate the activity of several membrane-bound phospholipases, including PLA 1 , and PLA 2 [45-52]. Previously, we showed that PlaF becomes active due to a dimer-to-monomer transition followed by tilting of the monomer in the membrane, resulting in $\mathrm{t}-\mathrm{PlaF}_{\mathrm{A}}$ being the active configuration of $\mathrm{PlaF}$ [19]. Here, we addressed the questions of how membrane-bound substrates reach the active site of $\mathrm{PlaF}_{\mathrm{A}}$ and how the characteristics of the active site tunnels determine the activity, specificity, and regioselectivity of $\mathrm{PlaF}$ for medium-chain substrates. We performed unbiased and biased MD simulations and showed by configurational free energy computations and mutational and enzymatic studies for $t-\mathrm{PlaF}_{A}$ that $\mathrm{A}$ ) access of the two main PlaF substrates DLPG and 2LMG occurs most likely through tunnel T2 in a tail-first mode, B) access of substrates with longer acyl chains or neutral head groups is less favorable, C) tail 1 access of DLPG and 2MLG in T2 is more favorable than tail 2 access, D) T3 accommodates the substrate tail to be hydrolyzed, and E) T1 and T3 are potential product egress pathways.

Previous studies indicated that the characteristics of substrate access tunnels can have a decisive influence on enzyme-substrate specificity and activity [53-56]. In t-PlaF, we focused on T1 and T2 because only these two allow direct access of GPL or LGPL substrates from the membrane in the $-\mathrm{PlaF}_{\mathrm{A}}$ configuration. By contrast, to enter into T3, substrates would need to pass through the solvent, which is energetically 
unfavorable. In di-PlaF, T2 is closest to the membrane with a distance of $7.4 \pm 1.5 \AA$ but T1 and T3 are at a distance $>12 \AA$ (Figure S18A). Hence, we also investigated substrate access to T2 in di-PlaF.

For assessing the energetics of substrate access, first, we generated 18 pathways, considering GPL and LGPL as substrates in T1 and T2 using SMD simulations. By relating the work along the reaction coordinate to the free energy difference between two states of the pulling simulations via Jarzynski's relation and considering the endpoint of the sMD trajectory closest to the Jarzynski average as the starting point for the next SMD simulation, we obtained low-free energy pathways of substrate access to the catalytic site. SMD simulations have been widely used to explore similar biological processes such as the loading of GPL substrates into human phospholipase $\mathrm{A}_{2}(\mathrm{PLA})$ [57] or recognition of arachidonic acid by cytochrome P450 2E1 across the access channel [58]. The pathways served for defining reference points for subsequent US simulations, such that distributions of sampled states sufficiently overlapped, which is essential to yield accurate results in PMF computations [59]. Applying US along pathways identified by SMD simulations [60] or targeted simulations [61, 62] has been shown to be an effective method of computing PMF. Moreover, the choice of an appropriate reaction coordinate is essential for this approach [63-65]. We probed for the convergence of our PMFs by comparing PMFs generated from increasing lengths of US simulations and found that US times of $\sim 300$ ns are needed to yield PMF differences below chemical accuracy [66]. Finally, we validated our PMF computations by comparing the computed absolute binding free energy of DLPG to PlaF for the most preferred access mode to an estimate of the experimental binding free energy.

The PMFs revealed that tail-first access through T2 is most preferred for DLPG and 2LMG. This finding is in line with the geometric analysis of T2, which revealed a tunnel bottleneck radius about half as large as the radius of DLPG deduced from the lipid's area-per-lipid, which can explain why a headgroup-first access is disfavorable for steric reasons. Furthermore, we showed that acyl chains of lipids embedded in a membrane can reach the interface region in unbiased MD simulations and, thus, can interact with 
567 the tunnel entrance. Such protrusions of lipid tails occur on a timescale of 568 approximately $100 \mathrm{~ns}$ depending on the extent of solvent exposure [67]. Tail-first 569 access of GPLs into the active site has also been found for cyclopropane fatty acid synthase [68]. Tail-first access through T2 is favored because of the predominant hydrophobic nature of the tunnel walls. By contrast, T1 contains a higher number of charged Asp and Arg residues and fewer neutral residues than T2, which makes tailfirst access there less favorable. In particular, the side chain of R80 protrudes into T1 at the tunnel entrance, which is reflected in an energy barrier of $\sim 3 \mathrm{kcal} \mathrm{mol}^{-1}$ found there for tail-first access.

Modifications in tunnels that connect a buried active site to the bulk solvent have been shown to affect ligand binding and unbinding [41]. Tunnel residues situated away from the active site are suitable targets for mutagenesis, as their replacement should not lead to a loss of the functionality of the active site [69]. Considering this, we introduced Trp substitutions to each of the three tunnels of PlaF and measured the activity of these PlaF variants. The Trp substitutions decreased PlaF's lipolytic activity for small and large substrates only when introduced in T2, which suggests that T2 is involved in substrate access. However, from such steady-state experiments, it cannot be excluded that the Trp substitutions influence product egress, too [54].

Among the investigated substrates, higher energy barriers for access to the active site were found for those with longer acyl chains and neutral head groups, concordant with PlaF's activity profile [19]. This finding may be explained with differences in the energetics of GPL self-assembly, which is influenced by the hydrocarbon chain length and the polarity of the head group [70]: Longer hydrocarbon chains and less polar head groups foster self-assembly, which would lead to higher energy barriers for leaving this equilibrium state [71] and entering into PlaF. These results indicate that the energetics of access of a membrane GPL substrate to the active site through tunnel T2 contributes to the substrate specificity of PlaF. 
energetically preferred over tail 2 access. If tail 1 enters first, the carbonyl oxy group at $\mathrm{C} 1$ of the glycero moiety can come closer to the nucleophilic $\mathrm{S} 137$ than if tail 2 enters first, (Figure S10) leading to preferential hydrolysis of the carboxylic ester bond at C1. Likewise, the regioselectivity of human 5-lipoxygenase is determined by the head/tailfirst type orientation of its main substrate arachidonic acid in the active site [72]: The arachidonic acid can be positioned in the holoenzyme active site with both head-first and tail-first orientation, but only the tail-first orientation results in a configuration that yields 5-lipoxygenating activity. These results indicate that the tail-first access mode of a diacyl GPL substrate determines the regioselectivity of PlaF for hydrolysis of the acyl chain bound to the sn-1 position.

As T3 is oriented to the membrane neither in the monomeric nor in the di-PlaF configuration, it likely does not contribute to substrate access. We suggest the role of T3 to accommodate the acyl chain of substrates before and products after hydrolysis. T3, with a length of $\sim 15 \AA$, provides adequate space for substrates with mediumlengths of acyl chains and, thus, may affect the specificity of PlaF. Substrate tunnels that accommodate acyl chains hydrolyzed from their respective precursors have also been described for cholesterol acyltransferases [73]. Likewise, lipid phosphate phosphatases harbor such a cavity, accommodating the substrate's acyl chain for optimal catalysis [74]. Site-directed mutagenesis in Candida rugosa lipase 1 revealed the role of such tunnels in determining the acyl chain length specificity [75].

As to di-PlaF, tail 1 access of DLPG across T2 revealed a free energy barrier of $\sim 13 \mathrm{kcal} \mathrm{mol}^{-1}$ (Figure S18B), in contrast to no free energy barrier in $\mathrm{t}-\mathrm{PlaF}_{\mathrm{A}}$ (Figure $5 B)$. This high barrier may arise because of the location of T2 in di-PlaF, $\sim 7 \AA$ above the membrane. Thus, substrates would need to pass through the solvent to enter T2. These findings indicate that di-PlaF is catalytically inactive, as determined experimentally [19], because of energetically unfavorable substrate access.

Our results from unbiased MD simulations of products suggest that $\mathrm{T} 1 \mathrm{and}$, to a lower extent, T3 are egress pathways of FAs. As to T1, this suggestion is in agreement with 
623 the crystal structure of PlaF, where FAs are found in T1 [19]. In the tilted orientation of 624 PlaF, FAs egressing via T1 would interact with the membrane interface and could 625 diffuse into it. FAs in a membrane can affect its fluidity and permeability and proteinlipid interactions, thereby regulating important cell processes including signal transduction, motility, and biofilm formation $[76,77]$. Via T3, they would egress into the periplasmic space. Anchored to the cytoplasmic membrane, PlaF is not a toxin targeting the host cell membrane but it has a direct influence on virulence adaptation of $P$. aeruginosa by modulating the membrane GPL composition [19]. However, it is unknown if FAs released from GPLs by PlaF are targeted to the external environment as for example diffusible FAs involved in cell-to-cell signaling $[17,18]$. In this case, egress of FAs via T3 to the periplasm and their further passive diffusion or active transport would be possible [78].

In summary, we identified T2 as the preferred tunnel for substrate access to t-PlaF $\mathrm{A}_{\mathrm{A}}$ while $\mathrm{T} 1$, and to a lesser extent T3, are likely egress routes for FAs. The energetically favorable tail 1 access of substrates is in agreement with PlaF's PLA function. The higher preference of PlaF for GPLs with medium-length acyl chains may be due to differences in the energetics of self-assembly and the length of $\mathrm{T} 3$, which accommodates them for hydrolysis (Figure 10). Finally, while t-PlaF $F_{A}$ enables substrate access to the active site, substrate access to di-PlaF is energetically unfavorable. Our results provide an atomistic-level understanding of the unique structural feature of $\mathrm{PlaF}$ that its function is dependent on monomerization followed by global reorientation of the single-pass TM protein at the membrane. They may furthermore aid in understanding the feedback regulation of PlaF, which is inhibited by FAs, and open up opportunities for developing potential drugs that inhibit PlaF to combat $P$. aeruginosa virulence during infections. 
A

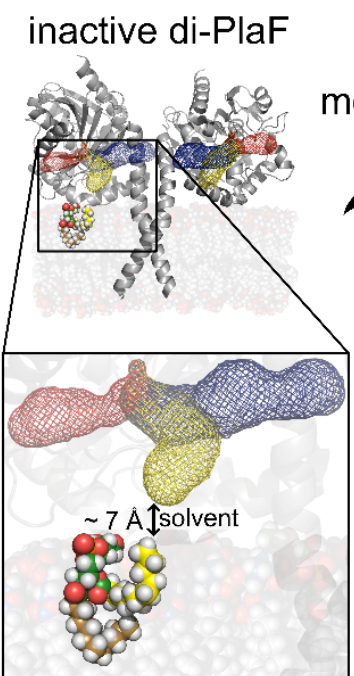

no hydrolysis
B active t-PlaFA
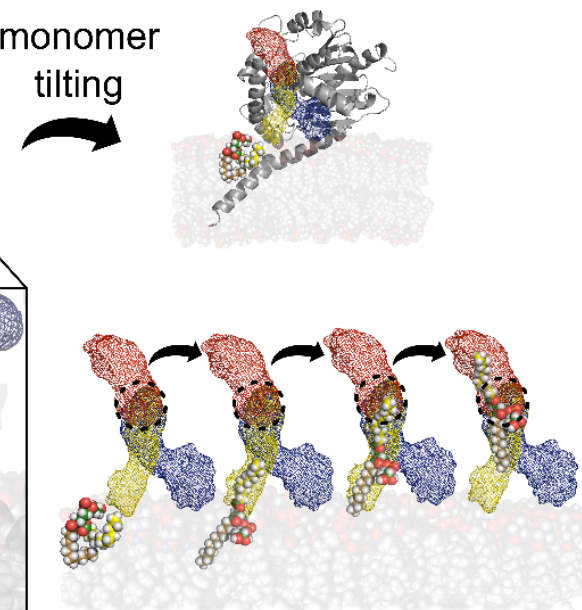

substrate hydrolysis
C

i)

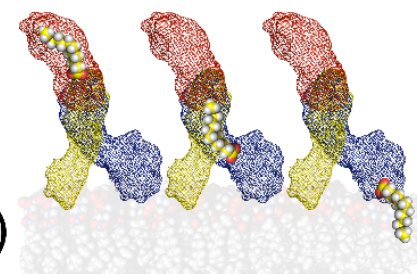

ii)

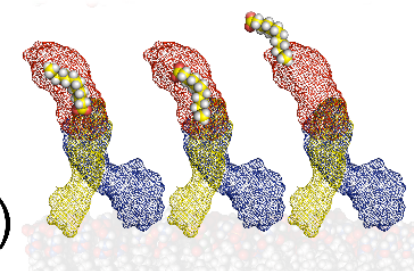

product egress
648

649

650

651

652

653

654

655

656

657

658

659

660

661

662

Figure 10: Schematic model of the mechanism of PlaF activity regulation. A) A higher concentration of PlaF results in the formation of an inactive dimer [19]. In di-PlaF, T2 is closer to the membrane than the other tunnels, but, still, the distance from the membrane interface is $\sim 7 \AA$, which requires the substrate to pass through the solvent. Hence, this configuration leads to inactive PlaF. B) At low concentrations, monomeric PlaF shows PLA $\mathrm{A}_{1}$ activity and adopts a tilted configuration [19]. In the tilted configuration, PlaF orients such that T1 and T2 come close to the membrane interface. Substrate access occurs via tail 1 into T2. The acyl chain reaches the active site (dashed black circle) of PlaF, but the $s n-1$ cleavage site is still away from the active site. Further loading of the acyl chain requires it to enter into T3, and the substrate is hydrolyzed. C) After hydrolysis, the FA is in T3. i) Now, either FA relocates into T1, with the carboxyl group towards the entrance. At the T1 entrance, FA can interact with the membrane interface and diffuse into it. ii) Alternatively, the FA can flip around, such that the carboxyl group faces the T3 entrance, from where it can exit into the periplasmic space.

\section{Materials and methods}

\section{Identification of the access tunnels}

Tunnels emerging from the active site of PlaF were identified using CAVER 3.0 [26]. The COM of the catalytic residues $\mathrm{S} 137$ and $\mathrm{H} 286$ was defined as the starting point of the search, from which the possible connections of the tunnels to the bulk solvent were identified. The catalytic residue D258 was not included in this search criteria since its side chain is distant from the catalytic site. Probe and shell radii of $2 \AA$ and $6 \AA$ were used, respectively. The probe radius of $2 \AA$ is slightly larger than the van der Waals 
671 radius of a phosphorous atom (i.e., $1.8 \AA$ ), present in every PlaF substrate to be 672 investigated.

\section{Starting structure preparation}

674 The crystal structure of PlaF is available from the Protein Data Bank (PDB) [79] (PDB 675 id: 6l8W) [19]. The first five residues of the C-terminus were missing in the structure 676 and, hence, were added using MODELLER [81]. The starting configuration of PlaF for

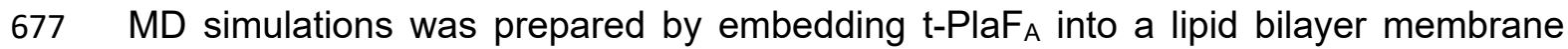
consisting of $75 \%$ DLPE and $25 \%$ DLPG. The tilted configuration of PlaF embedded in the membrane was predicted by the Positioning of Proteins in Membrane (PPM) method [82]. The head group composition of the membrane closely resembles that of the inner membrane of Gram-negative bacteria $[8,83,84]$. The prepared structure was used to investigate the loading mechanism of DLPG or DLPE into t-PlaF $F_{A}$. Furthermore, loading of DSPG and an LGPL, 2LMG, were also investigated. For that, t-PlaF $F_{A}$ was embedded into a membrane consisting of $\sim 10 \%$ of DSPG and 2 LMG in the upper leaflet. The GPL composition in the lower leaflet of these systems is the same as that used for investigating DLPG and DLPE. The systems were prepared and solvated using CHARMM/GUI [85] or PACKMOL-Memgen [86]. A distance of at least $15 \AA$ between the protein or membrane and the solvent box boundaries was used. To obtain a neutral system, counter ions were added that replaced solvent molecules. The size of the resulting systems was $\sim 140,000$ atoms. and one of the LGPL substrates (i.e., 2LMG), were also prepared to compare and decipher the energetics of lipid extraction from the membrane into solvent. Considering the orientation and position of $\mathrm{tPlaF}_{\mathrm{A}}$ in the membrane, one can safely assume that only substrates located in one leaflet will contact the catalytic domain of $t-\mathrm{PlaF}_{\mathrm{A}}$ and, hence, have direct access. Therefore, the composition of one leaflet was slightly modified to reflect the inclusion of the selected substrate. For this, a ratio in the upper leaflet of 6:2:1 for DLPE, DLPG, and the respective substrate was used. Using 
counter ions were added. The minimum water distance from the membrane surface to the solvent box boundaries was increased to $35 \AA$ to leave enough space between the substrate and the membrane surface and avoid interactions with periodic images during the extraction. Box dimensions in the $x$ and $y$ axes were set to $70 \AA$, resulting in systems comprised of $\sim 50,000$ atoms.

\section{Simulated extraction of substrates from the membrane}

MD simulations were performed using the GPU implementation of the AMBER 16 molecular simulation package $[87,88]$, employing the ff14SB force field for the protein [89], the Lipid17 force field for the lipids [90-92], and the TIP3P water model [93]. The SHAKE algorithm [94] was used to constrain bond lengths of hydrogen atoms to heavy atoms, enabling a time step of $2 \mathrm{fs}$. Long-range electrostatic interactions were considered using the Particle Mesh Ewald (PME) algorithm [95]. The system was energy-minimized by three mixed steepest descent/conjugate gradient calculations with a maximum of 20,000 steps each. First, the initial positions of the protein and membrane were restrained, followed by a calculation with restraints on the protein atoms only, and finalizing with a minimization without restraints. The minimized system was then gradually thermalized in two stages. Initially, the temperature was increased from $0 \mathrm{~K}$ to $100 \mathrm{~K}$ under NVT conditions, then from $100 \mathrm{~K}$ to $300 \mathrm{~K}$ under NPT conditions at 1 bar, using a Langevin thermostat [96]. The equilibration process continued for $5 \mathrm{~ns}$, before starting with production simulations. As usual in membrane MD simulations, the NPT ensemble was used, allowing the membrane to accommodate along the trajectory [97]. For US simulations, the pressure was maintained using an anisotropic Berendsen barostat [98], while for the rest of the simulations a semi-isotropic Berendsen barostat [98] was used, coupling the membrane $(x-y)$ plane with the constant-surface-tension dynamics. All analyses were performed by using CPPTRAJ [99]. Unless otherwise stated, molecular visualization was performed with Pymol [100] and VMD [101]. The Movie maker module within VMD was used to illustrate the acyl chain mobility and the access of substrates into PlaF.

To extract a substrate molecule from the membrane into one of the access tunnels, we 
selected the lipid that was closest to the entrance and pulled it from the membrane through the tunnel to the active site of PlaF, using constant velocity sMD simulations. Pulling simulations at low velocities are recommended for small polar molecules [102] and large lipids [103] to calculate free energy profiles. At the lowest pulling rates, lipids have time to adapt to energetically favorable conformations during the extraction process [103]. In a recent study investigating GPL binding to phospholipase A2 (PLA $)$, a constant pulling velocity of $5 \AA \mathrm{ns}^{-1}$ was used [57]. For the extraction of substrates, we considered all three possibilities by which a substrate may enter a tunnel: either the head group or one of the two tails. Depending on the type of head group (i.e., PG or $\mathrm{PE}$ ), each substrate was pulled by its oxygen or nitrogen atoms at a constant velocity of $1 \AA \mathrm{ns}^{-1}$ using a force constant of $5 \mathrm{kcal} \mathrm{mol}^{-1} \AA^{-2}$. When pulling at the tail, the terminal carbon atom of the respective acyl chain was used.

Each tunnel was divided into several segments connected through virtual points formed by the COM of amino acids lining the respective tunnel. The number of virtual points depends on the length and shape of the respective tunnel. The virtual points guided the extraction of substrates such that the substrates followed the path of the respective tunnel. In addition, to obtain a low energy pathway, an adaptive SMD protocol was implemented. For this, 50 replicas for each pulling simulation were carried out, and the work required was computed as a function of the reaction coordinate. The computed work was further related to free energy difference between two states of the pulling simulation applying Jarzynski's relation (eq. 1) [30].

$e^{-\Delta F / k T}=\overline{e^{-W / k T}}$

(Equation 1)

Here, $\Delta F$ is the free energy difference between two states, which is connected to work $W$ done on the system [30]. $k$ is the Boltzmann constant and $T$ the temperature of the system. The replica closest to the Jarzynski's average [30] was considered to describe the lowest-free-energy pathway and provided the starting point for the next pulling stage. Trajectories further away from that pathway were removed. This procedure results in faster convergence of PMF profiles, decreasing the overall computation needed [31]. 
For the systems without $t-P_{a} F_{A}$, the substrates were extracted with the same pulling velocity and spring constant, as mentioned above. However, to avoid edge effects, a substrate in the middle of the membrane was located. For this extraction process, the reaction coordinate was the distance between the head atom of the pulled substrate and $\mathrm{COM}$ of phosphorous atoms of the lipids in the opposite leaflet. Furthermore, to determine the free energy minimum of the phospholipids in the membrane more accurately, the substrate was first pulled into the membrane $(\sim 3 \AA)$, before pulling it out of the membrane

\section{Umbrella sampling and potential of mean force calculations}

To understand the substrate access mechanism in PlaF and to identify preferential substrate access tunnels, PMFs were computed based on US [34], taking structures from the SMD simulations as starting points. As a reaction coordinate, the COM distance of the three oxygen atoms of the glycerol moiety in the substrate to the COM of residues $\mathrm{S} 137$ and $\mathrm{H} 286$ (only $\mathrm{C} \alpha$ atoms) of the active site was used. This reaction coordinate was also taken for over all other systems for it describes the essential aspects of the structural transformation during substrate access. Consecutive positions of the substrates from the membrane to the active site as determined by pulling simulations were considered reference points for US, with each position corresponding to one umbrella window. To achieve sufficient overlap between the umbrella windows, distances between reference points of $\sim 1 \AA$ were used along the reaction coordinate. The length of individual tunnels and the size of acyl chains for respective substrates vary. Therefore, for sampling the access of different substrates, different numbers of windows were required for each tunnel. Selected positions of the lipid in the tunnel were restrained by harmonic potentials, using a force constant of 5 kcal mol-1 $\AA^{-2}$. To achieve sufficient convergence of the PMF profile, each window was sampled for $300 \mathrm{~ns}$, of which the last $100 \mathrm{~ns}$ were used to calculate the PMF. Distance values were recorded every 2 ps and processed with WHAM [35, 36]. To estimate the PMF error, the data was separated into blocks according to the maximum calculated autocorrelation time of 20 ns. The correlation time was obtained for the complete 
trajectory, excluding the first $20 \mathrm{~ns}$ of sampling data for equilibration. The last $100 \mathrm{~ns}$ of sampling data was split into five blocks of 20 ns each, a PMF profile was calculated for each block with WHAM, and the error at each PMF point was calculated as the standard error of the mean.

Similarly, for systems without t-PlaF $F_{A}$, trajectories obtained by pulling simulations were used to set up US simulations. Umbrella windows were extracted at distances of $1 \AA$ from the starting point of the pulling simulation until the substrate was not interacting with the membrane anymore. The selected positions of the lipid were restrained by harmonic potentials, using a force constant of $5 \mathrm{kcal} \mathrm{mol}^{-1} \AA^{-2}$ and as the reaction coordinate the distance of the COM of the three oxygen atoms of the glycerol moiety of the substrate to the COM of phosphorous atoms of the lower membrane leaflet. Each window was simulated for $100 \mathrm{~ns}$ at constant pressure (1 bar) and temperature $(300 \mathrm{~K})$ conditions until convergence was achieved. The first $20 \mathrm{~ns}$ of simulation data was discarded. WHAM $[35,36]$ was used to calculate the PMF. The PMFs were evaluated for convergence by checking the change in the free energy profile with the increase in sampling time at every $10 \mathrm{~ns}$. Furthermore, histograms of sampled configurations were visually inspected for sufficient overlap between the neighboring umbrella windows; otherwise, the iterative cycle in WHAM fails to converge and the free energy profiles have discontinuities.

\section{Absolute binding free energy from computed PMF}

The absolute binding free energy of substrates to PlaF was determined from the computed PMF using an approach modified from Chen and Kuyucak [104]. The PMF was integrated along the reaction coordinate (eq. 2) to calculate an association (equilibrium) constant $\left(K_{\text {eq }}\right)$.

$K_{e q}=\pi r^{2} \int_{\text {active_site }}^{\text {membrane }} e^{-W(\xi) / k T} d \xi$

(Equation 2)

Here, $r$ is the maximum bottleneck radius of the respective tunnel, which was determined by a CAVER analysis (Table 1 ), $\pi r^{2}$ is the cross-sectional area of the tunnel, $W(\xi)$ is the PMF at a specific value of the reaction coordinate, $k$ is the Boltzmann 
815 constant, and $T$ is the temperature at which the simulations were performed.

$816 K_{\text {eq }}$ was then transformed to the mole fraction scale $\left(K_{\mathrm{x}}\right)$, taking into account the 817 number of lipids $\left(N_{\mathrm{L}}\right)$ per surface area $A$ (eq. 3 ).

$818 K_{x}=K_{e q} \frac{N_{L}}{A}$

819 From $K_{\mathrm{x}}$, the difference in the free energy (eq. 4) between the bound and unbound 820 state $\left(\Delta G_{\mathrm{b}}^{\circ}\right)$ of a single substrate molecule was calculated.

$821 \Delta G_{\text {comp }}^{\circ}=-R T \ln \left(K_{x}\right)$

(Equation 4)

822

\section{Blocking access of the PlaF substrates}

To corroborate predicted access tunnels for PlaF substrates, we intended to block these by small-to-tryptophan substitutions of tunnel-lining residues. To do so, we identified possible substitution sites from our previous CAVER analyses, taking into account the tunnels' bottleneck radii and lengths. For these analyses, the same trajectory used to search for tunnels in t-PlaF $\mathrm{A}$ was considered. Finally, 4-5 amino acids within each tunnel were selected for substitutions.

In the first step, all the amino acids except glycines and prolines within $3 \AA$ of individual tunnels and oriented towards a tunnel were considered. In turn, residues with an outward orientation were disregarded as a substitution there will likely not block the tunnel. Furthermore, as the TM and JM helix was found to be important for both the dimerization and the activity of PlaF [19], residues of these helices were excluded. Finally, the catalytic residues S137, D258, and H286 and other residues of the active site were disregarded to avoid affecting the activity of PlaF.

The selected residues of each tunnel were substituted to tryptophan using FoldX [105], and the stability of the Plaf variants was evaluated in terms of the change in free energy $(\Delta \Delta G)$ with respect to the wild type [106]. Single amino acid substitutions were performed 10 times for each proposed residue of each tunnel, and the results were averaged. If the average $\Delta \Delta G>3 \mathrm{kcal} \mathrm{mol}^{-1}$, the substitution is considered destabilizing [107] and was not further pursued. To check if the proposed substitutions 
842 will block the tunnel, the bottleneck radius of the variant tunnels was recalculated using

843 CAVER. As done earlier, the probe radius was set to $2 \AA$. If no tunnel was identified 844 with this criterium, the probe radius for tunnel search was reduced until the tunnels started to appear again.

\section{Biological evaluation of PlaF activity from mutations}

a) Site-directed mutagenesis, protein expression, and purification

The plasmids for expression of PlaF variants with substitutions in the tunnels (Table S5) were created by PCR, using Phusion DNA polymerase (Thermo Fischer Scientific) in whole plasmid amplification, with mutagenic oligonucleotides (Table S7) designed for the SLIC method [108], and p-plaF plasmid [19] as a template. The presence of desired nucleotide substitutions was confirmed by DNA sequencing (MWG Biotech, Ebersberg, Germany). PlaF was purified from $P$. aeruginosa p-plaF membranes and solubilized with DDM, as described previously [109]. Proteins were analyzed by polyacrylamide gel electrophoresis under denaturation conditions (SDS-PAGE) on 14\% (w/v) gels, as described by Laemmli [110]. The protein concentration was determined by measuring the $A_{280 \mathrm{~nm}}$ using a NanoDrop $2000 \mathrm{C}$ spectrophotometer (Thermo Fisher Scientific Inc., Waltham, Massachusetts, USA). The extinction coefficients for PlaF and the variants were calculated with the ProtParam tool (Navia-Paldanius et al., 2012), considering the amino acid exchange and a $\mathrm{His}_{6}$-tag.

\section{b) Enzyme activity assays and kinetic studies}

The esterase activities of PlaF and variants were determined with $p$-NPB as substrate as described previously [111], using a 96-well microplate and starting the reaction by adding $100 \mu \mathrm{l}$ of PlaF sample $(16 \mathrm{nM})$ to the $100 \mu \mathrm{l}$ of $p$-NPB solution $(2 \mathrm{mM})$. Kinetic parameters $\left(K_{\mathrm{m}}\right.$ and $\left.k_{\text {cat }}\right)$ for hydrolysis of $p$-NPB were determined using $8 \mathrm{nM}$ enzyme as described previously [109]. Kinetic parameters were determined by non-linear regression analysis of data fitted to the Michaelis-Menten equation with PrismLab. Enzyme activities with DLPG and LGPLs were determined according to the established protocol in ref. [112]. For enzymatic reactions, $25 \mu \mathrm{L}$ of PlaF or the variant $(16 \mathrm{nM})$ and $25 \mu \mathrm{L}$ of DLPG solution were used. The amount of released FAs after $24 \mathrm{~h}$ 
871 of reaction were calculated from the calibration curve using oleic acid at concentrations 872 ranging from 0.1 to $1.0 \mathrm{mM}$.

$874 \mathrm{PlaF}$ and variants $(28.1 \mu \mathrm{M}, 10 \mu \mathrm{L})$ loaded into the measuring capillaries (Prometheus NT.Plex nanoDSF Grade Standard Capillary Chips) were heated from 20 to $90{ }^{\circ} \mathrm{C}$ $\left(1^{\circ} \mathrm{C} \mathrm{min}-1\right.$ heating rate), and the intrinsic protein fluorescence was recorded at $330 \mathrm{~nm}$ and $350 \mathrm{~nm}$ using the Prometheus NT.Plex nanoDSF device (Nano Temper, Munich, Germany) [113]. The melting points were calculated from the first derivative of the ratio of $F_{350 \mathrm{~nm}}$ and $F_{330 \mathrm{~nm}}$ using the PR.ThermControl software (Nano Temper, Munich, Germany) [113].

\section{Egress of PlaF products}

882

To determine the egress pathways of PlaF products, a system with 2LMG substrate was considered. The final snapshot at 300 ns of the US simulations of the window with the substrate close to the active site was considered as the starting structure for unbiased MD simulations. 2LMG was cleaved into the products: MYR and PGR, without altering the orientation of each product within the tunnels. Atomic partial charges for the products were derived according to the restraint electrostatic potential fit (RESP) procedure [114], as implemented in Antechamber [115]. Geometry optimizations and subsequent single-point calculations were performed with Gaussian [116] at the Hartree-Fock (HF) level with the $6-31 G^{*}$ basis set. Force field parameters for the products were taken from the general amber force field for organic molecules (GAFF, version 2) [117]. The prepared system was then minimized, thermalized, and equilibrated using the protocol described above for MD simulations. 12 replicas of production MD simulations of $3 \mu$ s length each under NPT conditions were performed. The distance of the 2LMG products to the entrance of each tunnel was computed for each replica. 
898

899

900

901

902

903

904

905

906

907

908

909

910

911

912

913

914

915

916

917

\section{Acknowledgments}

This study was funded by the Deutsche Forschungsgemeinschaft (DFG, German Research Foundation) project no. 267205415 / CRC 1208 grant to FK and KEJ (TP 02) as well as HG (TP 03). We are grateful for computational support by the "Zentrum für Informations und Medientechnologie" at the Heinrich-Heine-Universität Düsseldorf and the computing time provided by the John von Neumann Institute for Computing (NIC) to HG on the supercomputer JUWELS at Jülich Supercomputing Centre (JSC) (user IDs: HDD18; plaf).

\section{Author contributions}

H.G. conceptualization, supervision, analysis, visualization, writing; F.K. conceptualization, supervision, analysis, visualization, writing; K.-E.J. supervision, writing; S.A. investigation, analysis, visualization, writing; C.H.S. investigation, analysis, visualization, writing; S.N.S.V. analysis, visualization, writing.

\section{Competing interests}

The authors declare no competing interests.

\section{Additional information}

Supplementary information is available for this paper. 


\section{References}

1. Chahtane, H., Nogueira Fuller, T., Allard, P. M., Marcourt, L., Ferreira Queiroz, E., Shanmugabalaji, V., Falquet, J., Wolfender, J. L. \& Lopez-Molina, L. (2018) The plant pathogen Pseudomonas aeruginosa triggers a DELLA-dependent seed germination arrest in Arabidopsis, eLife 7, 1-34.

2. Tacconelli, E., Carrara, E., Savoldi, A., Harbarth, S., Mendelson, M., Monnet, D. L., Pulcini, C., Kahlmeter, G., Kluytmans, J., Carmeli, Y., Ouellette, M., Outterson, K., Patel, J., Cavaleri, M., Cox, E. M., Houchens, C. R., Grayson, M. L., Hansen, P., Singh, N., Theuretzbacher, U., Magrini, N. \& Group, W. H. O. P. P. L. W. (2018) Discovery, research, and development of new antibiotics: the WHO priority list of antibiotic-resistant bacteria and tuberculosis, Lancet Infect Dis. 18, 318-327.

3. Gellatly, S. L. \& Hancock, R. E. W. (2013) Pseudomonas aeruginosa: new insights into pathogenesis and host defenses, Pathog Dis. 67, 159-173.

4. da Mata Madeira, P. V., Zouhir, S., Basso, P., Neves, D., Laubier, A., Salacha, R., Bleves, S., Faudry, E., Contreras-Martel, C. \& Dessen, A. (2016) Structural Basis of Lipid Targeting and Destruction by the Type V Secretion System of Pseudomonas aeruginosa, J Mol Biol. 428, $1790-803$.

5. Salacha, R., Kovacic, F., Brochier-Armanet, C., Wilhelm, S., Tommassen, J., Filloux, A., Voulhoux, R. \& Bleves, S. (2010) The Pseudomonas aeruginosa patatin-like protein PIpD is the archetype of a novel Type V secretion system, Environ Microbiol. 12, 1498-512.

6. Fozo, E. M. \& Rucks, E. A. (2016) The Making and Taking of Lipids: The Role of Bacterial Lipid Synthesis and the Harnessing of Host Lipids in Bacterial Pathogenesis, Adv Microb Physiol. 69, 51-155.

7. Housley, N. A., Winkler, H. H. \& Audia, J. P. (2011) The Rickettsia prowazekii ExoU homologue possesses phospholipase A1 (PLA1), PLA2, and lyso-PLA2 activities and can function in the absence of any eukaryotic cofactors in vitro, $J$ Bacteriol. 193, 4634-42.

8. Dowhan, W. (1997) Molecular basis for membrane phospholipid diversity: why are there so many lipids?, Annu Rev Biochem. 66, 199-232.

9. Tannaes, T., Bukholm, I. K. \& Bukholm, G. (2005) High relative content of lysophospholipids of Helicobacter pylori mediates increased risk for ulcer disease, FEMS Immunol Med Microbiol. 44, 17-23.

10. Zheng, L., Lin, Y., Lu, S., Zhang, J. \& Bogdanov, M. (2017) Biogenesis, transport and remodeling of lysophospholipids in Gram-negative bacteria, Biochim Biophys Acta Mol Cell Biol Lipids. 1862, 1404-1413. 
11. Corradi, V., Sejdiu, B. I., Mesa-Galloso, H., Abdizadeh, H., Noskov, S. Y., Marrink, S. J. \& Tieleman, D. P. (2019) Emerging Diversity in Lipid-Protein Interactions, Chem Rev. 119, 5775-5848.

12. Koehler, J., Sulistijo, E. S., Sakakura, M., Kim, H. J., Ellis, C. D. \& Sanders, C. R. (2010) Lysophospholipid micelles sustain the stability and catalytic activity of diacylglycerol kinase in the absence of lipids, Biochemistry 49, 7089-99.

13. Nomura, T., Cranfield, C. G., Deplazes, E., Owen, D. M., Macmillan, A., Battle, A. R., Constantine, M., Sokabe, M. \& Martinac, B. (2012) Differential effects of lipids and lyso-lipids on the mechanosensitivity of the mechanosensitive channels MscL and MscS, Proc Natl Acad Sci U S A 109, 8770-5.

14. Le Senechal, C., Crouzet, M., Costaglioli, P., Barthe, C., Bure, C. \& Vilain, S. (2019) Phospholipid Content of Pseudomonas aeruginosa PAO1 Is Modulated by the Growth Phase Rather Than the Immobilization State, Lipids 54, 519-529.

15. Benamara, H., Rihouey, C., Abbes, I., Ben Mlouka, M. A., Hardouin, J., Jouenne, T. \& Alexandre, S. (2014) Characterization of membrane lipidome changes in Pseudomonas aeruginosa during biofilm growth on glass wool, PLoS One. 9, e108478.

16. Zhou, L., Zhang, L. H., Camara, M. \& He, Y. W. (2017) The DSF Family of Quorum Sensing Signals: Diversity, Biosynthesis, and Turnover, Trends Microbiol. 25, 293-303.

17. Twomey, K. B., O'Connell, O. J., McCarthy, Y., Dow, J. M., O'Toole, G. A., Plant, B. J. \& Ryan, R. P. (2012) Bacterial cis-2-unsaturated fatty acids found in the cystic fibrosis airway modulate virulence and persistence of Pseudomonas aeruginosa, Isme J. 6, 939-950.

18. Ryan, R. P., Fouhy, Y., Garcia, B. F., Watt, S. A., Niehaus, K., Yang, L., Tolker-Nielsen, T. \& Dow, J. M. (2008) Interspecies signalling via the Stenotrophomonas maltophilia diffusible signal factor influences biofilm formation and polymyxin tolerance in Pseudomonas aeruginosa, Mol Microbiol. 68, 75-86.

19. Bleffert, F., Granzin, J., Caliskan, M., Schott-Verdugo, S., Siebers, M., Thiele, B., Rahme, L., Felgner, S., Doermann, P., Gohlke, H., Batra-Safferling, R., Jaeger, K.-E. \& Kovacic, F. (2021) Evidence for a bacterial Lands cycle phospholipase A: Structural and mechanistic insights into membrane phospholipid remodeling, bioRxiv, 2021.06.22.448587.

20. Gendrin, C., Contreras-Martel, C., Bouillot, S., Elsen, S., Lemaire, D., Skoufias, D. A., Huber, P., Attree, I. \& Dessen, A. (2012) Structural basis of cytotoxicity mediated by the type III secretion toxin ExoU from Pseudomonas aeruginosa, PLoS Pathog. 8, e1002637.

21. Murayama, K., Kano, K., Matsumoto, Y. \& Sugimori, D. (2013) Crystal structure of phospholipase A1 from Streptomyces albidoflavus NA297, J Struct Biol. 182, 192-6.

22. Tiesinga, J. J., van Pouderoyen, G., Nardini, M., Ransac, S. \& Dijkstra, B. W. (2007) Structural basis of phospholipase activity of Staphylococcus hyicus lipase, J Mol Biol. 371, 44756. 
23. Scandella, C. J. \& Kornberg, A. (1971) A membrane-bound phospholipase A1 purified from Escherichia coli, Biochemistry 10, 4447-56.

24. Snijder, H. J., Kingma, R. L., Kalk, K. H., Dekker, N., Egmond, M. R. \& Dijkstra, B. W. (2001) Structural investigations of calcium binding and its role in activity and activation of outer membrane phospholipase A from Escherichia coli, J Mol Biol. 309, 477-89.

25. Schmidt, D., Boehm, M., McClendon, C. L., Torella, R. \& Gohlke, H. (2019) CosolventEnhanced Sampling and Unbiased Identification of Cryptic Pockets Suitable for StructureBased Drug Design, J Chem Theory Comput. 15, 3331-3343.

26. Chovancova, E., Pavelka, A., Benes, P., Strnad, O., Brezovsky, J., Kozlikova, B., Gora, A., Sustr, V., Klvana, M., Medek, P., Biedermannova, L., Sochor, J. \& Damborsky, J. (2012) CAVER 3.0: a tool for the analysis of transport pathways in dynamic protein structures, PLOS Comput Biol. 8, e1002708.

27. Goldstein, D. A. \& Solomon, A. K. (1960) Determination of equivalent pore radius for human red cells by osmotic pressure measurement, J Gen Physiol. 44, 1-17.

28. Pan, J., Marquardt, D., Heberle, F. A., Kucerka, N. \& Katsaras, J. (2014) Revisiting the bilayer structures of fluid phase phosphatidylglycerol lipids: Accounting for exchangeable hydrogens, Biochim Biophys Acta. 1838, 2966-9.

29. Izrailev, S., Stepaniants, S., Isralewitz, B., Kosztin, D., Lu, H., Molnar, F., Wriggers, W. \& Schulten, K. (1999). Steered Molecular Dynamics. Deuflhard P., Hermans J., Leimkuhler B., Mark A.E., Reich S., Skeel R.D. (Eds), Computational Molecular Dynamics: Challenges, Methods, Ideas. Lecture Notes in Computational Science and Engineering, vol 4. Springer, Berlin, Heidelberg, Germany.

30. Jarzynski, C. (1997) Nonequilibrium equality for free energy differences, Phys Rev Lett. 78, 2690-2693.

31. Bureau, H. R., Merz, D. R., Jr., Hershkovits, E., Quirk, S. \& Hernandez, R. (2015) Constrained Unfolding of a Helical Peptide: Implicit versus Explicit Solvents, PLoS One. 10, e0127034.

32. Homeyer, N., Stoll, F., Hillisch, A. \& Gohlke, H. (2014) Binding Free Energy Calculations for Lead Optimization: Assessment of Their Accuracy in an Industrial Drug Design Context, $J$ Chem Theory Comput. 10, 3331-3344.

33. Izrailev, S., Crofts, A. R., Berry, E. A. \& Schulten, K. (1999) Steered molecular dynamics simulation of the Rieske subunit motion in the cytochrome bc(1) complex, Biophys J. 77, 175368.

34. Torrie, G. M. \& Valleau, J. P. (1977) Nonphysical sampling distributions in Monte Carlo free-energy estimation: Umbrella sampling, J Comput Phys. 23, 187-199. 
35. Kumar, S., Bouzida, D., Swendsen, R. H., Kollman, P. A. \& Rosenberg, J. M. (1992) The Weighted Histogram Analysis Method for Free-Energy Calculations on Biomolecules .1. The Method, J Comput Chem. 13, 1011-1021.

36. Grossfield, A. (2016) WHAM: the weighted histogram analysis method, version 2.0.9. Available online: http://membrane.urmc.rochester.edu/content/wham (accessed on 21 June 2021).

37. Bisswanger, H. (2017) Enzyme Kinetics: Principles and Methods, $3^{\text {rd }}$ edn., Wiley-VCH, Weinheim, Germany.

38. Nelson, D. L., Lehninger, A. L. \& Cox, M. M. (2008) Lehninger Principles of Biochemistry, $5^{\text {th }}$ edn., W.H. Freeman, New York, USA.

39. Strunk, C. H. (2020) Biochemische Charakterisierung der Phospholipase PlaF aus Pseudomonas aeruginosa und ihres humanen Homologs ABHD6, PhD Thesis, Heinrich Heine University, Düsseldorf, Germany.

40. Michel, J. \& Essex, J. W. (2010) Prediction of protein-ligand binding affinity by free energy simulations: assumptions, pitfalls and expectations, J Comput Aided Mol Des. 24, 639-58.

41. Biedermannova, L., Prokop, Z., Gora, A., Chovancova, E., Kovacs, M., Damborsky, J. \& Wade, R. C. (2012) A single mutation in a tunnel to the active site changes the mechanism and kinetics of product release in haloalkane dehalogenase LinB, J Biol Chem. 287, 29062-74.

42. Friedman, R., Nachliel, E. \& Gutman, M. (2005) Molecular dynamics of a protein surface: ion-residues interactions, Biophys J. 89, 768-81.

43. Salamanca Viloria, J., Allega, M. F., Lambrughi, M. \& Papaleo, E. (2017) An optimal distance cutoff for contact-based Protein Structure Networks using side-chain centers of mass, Sci Rep. 7, 2838.

44. Cornell, C. E., Black, R. A., Xue, M., Litz, H. E., Ramsay, A., Gordon, M., Mileant, A., Cohen, Z. R., Williams, J. A., Lee, K. K., Drobny, G. P. \& Keller, S. L. (2019) Prebiotic amino acids bind to and stabilize prebiotic fatty acid membranes, Proc Natl Acad Sci U S A 116, 17239-17244.

45. Awasthi, M., Batra, J. \& Kateriya, S. (2012) Disulphide bridges of phospholipase C of Chlamydomonas reinhardtii modulates lipid interaction and dimer stability, PLoS One. 7 , e39258.

46. de Oliveira, A. H., Giglio, J. R., Andriao-Escarso, S. H., Ito, A. S. \& Ward, R. J. (2001) A $\mathrm{pH}$-induced dissociation of the dimeric form of a lysine 49-phospholipase A2 abolishes Ca2+independent membrane damaging activity, Biochemistry 40, 6912-20.

47. Dekker, N., Tommassen, J., Lustig, A., Rosenbusch, J. P. \& Verheij, H. M. (1997) Dimerization regulates the enzymatic activity of Escherichia coli outer membrane phospholipase A, J Biol Chem. 272, 3179-84. 
48. Gomes, A. A. S., Cardoso, F. F., Souza, M. F., Oliveira, C. L. P., Perahia, D., Magro, A. J. \& Fontes, M. R. M. (2020) The allosteric activation mechanism of a phospholipase A2-like toxin from Bothrops jararacussu venom: a dynamic description, Sci Rep. 10, 16252.

49. Kingma, R. L. \& Egmond, M. R. (2002) Activation of a covalent outer membrane phospholipase A dimer, Eur J Biochem. 269, 2178-85.

50. Malley, K. R., Koroleva, O., Miller, I., Sanishvili, R., Jenkins, C. M., Gross, R. W. \& Korolev, S. (2018) The structure of $\mathrm{iPLA}_{2} \beta$ reveals dimeric active sites and suggests mechanisms of regulation and localization, Nat Commun. 9, 765.

51. Snijder, H. J., Ubarretxena-Belandia, I., Blaauw, M., Kalk, K. H., Verheij, H. M., Egmond, M. R., Dekker, N. \& Dijkstra, B. W. (1999) Structural evidence for dimerization-regulated activation of an integral membrane phospholipase, Nature 401, 717-721.

52. Tomasselli, A. G., Hui, J., Fisher, J., Zürcher-Neely, H., Reardon, I. M., Oriaku, E., Kézdy, F. J. \& Heinrikson, R. L. (1989) Dimerization and activation of porcine pancreatic phospholipase A2 via substrate level acylation of lysine 56, J Biol Chem. 264, 10041-10047.

53. Kokkonen, P., Beier, A., Mazurenko, S., Damborsky, J., Bednar, D. \& Prokop, Z. (2021) Substrate inhibition by the blockage of product release and its control by tunnel engineering, RSC Chem Biol. 2, 645-655.

54. Kokkonen, P., Slanska, M., Dockalova, V., Pinto, G. P., Sanchez-Carnerero, E. M., Damborsky, J., Klan, P., Prokop, Z. \& Bednar, D. (2020) The impact of tunnel mutations on enzymatic catalysis depends on the tunnel-substrate complementarity and the rate-limiting step, Comput Struct Biotechnol J. 18, 805-813.

55. Lu, Z., Li, X., Zhang, R., Yi, L., Ma, Y. \& Zhang, G. (2019) Tunnel engineering to accelerate product release for better biomass-degrading abilities in lignocellulolytic enzymes, Biotechnol Biofuels. 12, 275.

56. Wullich, S. C., Wijma, H. J., Janssen, D. B. \& Fetzner, S. (2021) Stabilizing AqdC, a Pseudomonas Quinolone Signal-Cleaving Dioxygenase from Mycobacteria, by FRESCOBased Protein Engineering, Chembiochem. 22, 733-742.

57. Mouchlis, V. D., Bucher, D., McCammon, J. A. \& Dennis, E. A. (2015) Membranes serve as allosteric activators of phospholipase A2, enabling it to extract, bind, and hydrolyze phospholipid substrates, Proc Natl Acad Sci U S A 112, E516-25.

58. Cui, Y. L., Zheng, Q. C., Zhang, J. L. \& Zhang, H. X. (2015) Molecular basis of the recognition of arachidonic acid by cytochrome P450 2E1 along major access tunnel, Biopolymers 103, 53-66.

59. Wu, D. (2010) An efficient umbrella potential for the accurate calculation of free energies by molecular simulation, J Chem Phys. 133, 044115. 
60. Yang, Y., Pan, L., Lightstone, F. C. \& Merz, K. M., Jr. (2016) The Role of Molecular Dynamics Potential of Mean Force Calculations in the Investigation of Enzyme Catalysis, Methods Enzymol. 577, 1-29.

61. Ahmed, A., Rippmann, F., Barnickel, G. \& Gohlke, H. (2011) A normal mode-based geometric simulation approach for exploring biologically relevant conformational transitions in proteins, J Chem Inf Model. 51, 1604-22.

62. Ciupka, D. \& Gohlke, H. (2017) On the potential alternate binding change mechanism in a dimeric structure of Pyruvate Phosphate Dikinase, Sci Rep. 7, 8020.

63. Domanski, J., Hedger, G., Best, R. B., Stansfeld, P. J. \& Sansom, M. S. P. (2017) Convergence and Sampling in Determining Free Energy Landscapes for Membrane Protein Association, J Phys Chem B. 121, 3364-3375.

64. Rosta, E., Woodcock, H. L., Brooks, B. R. \& Hummer, G. (2009) Artificial reaction coordinate "tunneling" in free-energy calculations: the catalytic reaction of RNase $\mathrm{H}, \mathrm{J}$ Comput Chem. 30, 1634-41.

65. Neale, C. \& Pomes, R. (2016) Sampling errors in free energy simulations of small molecules in lipid bilayers, Biochim Biophys Acta. 1858, 2539-2548.

66. Waschenbach, L., Gertzen, C. G. W., Keitel, V. \& Gohlke, H. (2020) Dimerization energetics of the G-protein coupled bile acid receptor TGR5 from all-atom simulations, $J$ Comput Chem. 41, 874-884.

67. Tahir, M. A., Van Lehn, R. C., Choi, S. H. \& Alexander-Katz, A. (2016) Solvent-exposed lipid tail protrusions depend on lipid membrane composition and curvature, Biochim Biophys Acta. 1858, 1207-15.

68. Hari, S. B., Grant, R. A. \& Sauer, R. T. (2018) Structural and Functional Analysis of E. coli Cyclopropane Fatty Acid Synthase, Structure 26, 1251-1258 e3.

69. Pavlova, M., Klvana, M., Prokop, Z., Chaloupkova, R., Banas, P., Otyepka, M., Wade, R. C., Tsuda, M., Nagata, Y. \& Damborsky, J. (2009) Redesigning dehalogenase access tunnels as a strategy for degrading an anthropogenic substrate, Nat Chem Biol. 5, 727-733.

70. King, M. D. \& Marsh, D. (1987) Head group and chain length dependence of phospholipid self-assembly studied by spin-label electron spin resonance, Biochemistry 26, 1224-31.

71. Tieleman, D. P. \& Marrink, S. J. (2006) Lipids out of equilibrium: energetics of desorption and pore mediated flip-flop, J Am Chem Soc. 128, 12462-7.

72. Saura, P., Marechal, J. D., Masgrau, L., Lluch, J. M. \& Gonzalez-Lafont, A. (2016) Computational insight into the catalytic implication of head/tail-first orientation of arachidonic acid in human 5-lipoxygenase: consequences for the positional specificity of oxygenation, Phys Chem Chem Phys. 18, 23017-35. 
73. Qian, H., Zhao, X., Yan, R., Yao, X., Gao, S., Sun, X., Du, X., Yang, H., Wong, C. C. L. \& Yan, N. (2020) Structural basis for catalysis and substrate specificity of human ACAT1, Nature 581, 333-338.

74. Tong, S., Lin, Y., Lu, S., Wang, M., Bogdanov, M. \& Zheng, L. (2016) Structural Insight into Substrate Selection and Catalysis of Lipid Phosphate Phosphatase PgpB in the Cell Membrane, J Biol Chem. 291, 18342-52.

75. Schmitt, J., Brocca, S., Schmid, R. D. \& Pleiss, J. (2002) Blocking the tunnel: engineering of Candida rugosa lipase mutants with short chain length specificity, Protein Eng. 15, 595-601.

76. Ibarguren, M., Lopez, D. J. \& Escriba, P. V. (2014) The effect of natural and synthetic fatty acids on membrane structure, microdomain organization, cellular functions and human health, Biochim Biophys Acta. 1838, 1518-28.

77. Desbois, A. P. \& Smith, V. J. (2010) Antibacterial free fatty acids: activities, mechanisms of action and biotechnological potential, Appl Microbiol Biotechnol. 85, 1629-42.

78. Liu, H., Yu, C., Feng, D., Cheng, T., Meng, X., Liu, W., Zou, H. \& Xian, M. (2012) Production of extracellular fatty acid using engineered Escherichia coli, Microb Cell Fact. 11, 41.

79. Berman, H. M., Westbrook, J., Feng, Z., Gilliland, G., Bhat, T. N., Weissig, H., Shindyalov, I. N. \& Bourne, P. E. (2000) The Protein Data Bank, Nucleic Acids Res. 28, 235-42.

80. Bleffert, F., Granzin, J., Caliskan, M., Schott-Verdugo, S.N., Rahme, L., Siebers, M., Thiele, B., Doermann, P., Gohlke, H., Batra-Safferling, R., Kovacic, F., Jaeger, K.-E. (2019) Structural and mechanistic insights into phospholipase A-mediated membrane phospholipid degradation associated with bacterial virulence. PDB ID: 6I8W, DOI: 10.2210/pdb6I8W/pdb.

81. Sali, A. \& Blundell, T. L. (1993) Comparative protein modelling by satisfaction of spatial restraints, J Mol Biol. 234, 779-815.

82. Lomize, M. A., Pogozheva, I. D., Joo, H., Mosberg, H. I. \& Lomize, A. L. (2012) OPM database and PPM web server: resources for positioning of proteins in membranes, Nucleic Acids Res. 40, D370-6.

83. Conrad, R. S. \& Gilleland, H. E., Jr. (1981) Lipid alterations in cell envelopes of polymyxinresistant Pseudomonas aeruginosa isolates, J Bacteriol. 148, 487-97.

84. Murzyn K, R. T., Pasenkiewicz-Gierula M (2005) Phosphatidylethanolaminephosphatidylglycerol bilayer as a model of the inner bacterial membrane, Biophys J. 88(2), 1091-1103.

85. Jo, S., Lim, J. B., Klauda, J. B. \& Im, W. (2009) CHARMM-GUI Membrane Builder for mixed bilayers and its application to yeast membranes, Biophys J. 97, 50-8. 
86. Schott-Verdugo, S. \& Gohlke, H. (2019) PACKMOL-Memgen: A Simple-To-Use, Generalized Workflow for Membrane-Protein-Lipid-Bilayer System Building, J Chem Inf Model. 59, 2522-2528.

87. Case, D. A., Cheatham, T. E., 3rd, Darden, T., Gohlke, H., Luo, R., Merz, K. M., Jr., Onufriev, A., Simmerling, C., Wang, B. \& Woods, R. J. (2005) The Amber biomolecular simulation programs, J Comput Chem. 26, 1668-1688.

88. Le Grand, S., Gotz, A. W. \& Walker, R. C. (2013) SPFP: Speed without compromise-A mixed precision model for GPU accelerated molecular dynamics simulations, Comput Phys Commun. 184, 374-380.

89. Maier, J. A., Martinez, C., Kasavajhala, K., Wickstrom, L., Hauser, K. E. \& Simmerling, C. (2015) ff14SB: Improving the Accuracy of Protein Side Chain and Backbone Parameters from ff99SB, J Chem Theory Comput. 11, 3696-713.

90. D.A. Case, D. S. C., T.E. Cheatham, III, T.A. Darden, R.E. Duke, T.J. Giese, H. Gohlke, A.W. Goetz, D.Greene,N. Homeyer, S. Izadi, A. Kovalenko, T.S. Lee, S. LeGrand, P. Li, C. Lin, J. Liu, T. Luchko, R. Luo, D. Mermelstein, K.M. Merz, G. Monard, H. Nguyen, I. Omelyan, A. Onufriev, F. Pan, R. Qi, D.R. Roe, A. Roitberg, C. Sagui, C.L. Simmerling, W.M. Botello-Smith, J. Swails, R.C. Walker, J. Wang, R.M. Wolf, X. Wu, L. Xiao, D.M. York and P.A. Kollman (2017) AMBER 2017 Reference Manual, University of California, San Francisco.

91. Dickson, C. J., Madej, B. D., Skjevik, A. A., Betz, R. M., Teigen, K., Gould, I. R. \& Walker, R. C. (2014) Lipid14: The Amber Lipid Force Field, J Chem Theory Comput. 10, 865-879.

92. Skjevik, A. A., Madej, B. D., Dickson, C. J., Lin, C., Teigen, K., Walker, R. C. \& Gould, I. R. (2016) Simulation of lipid bilayer self-assembly using all-atom lipid force fields, Phys Chem Chem Phys. 18, 10573-84.

93. Jorgensen, W. L., Chandrasekhar, J., Madura, J. D., Impey, R. W. \& Klein, M. L. (1983) Comparison of Simple Potential Functions for Simulating Liquid Water, J Chem Phys. 79, 926935.

94. Ryckaert, J. P., Ciccotti, G. \& Berendsen, H. J. C. (1977) Numerical-Integration of Cartesian Equations of Motion of a System with Constraints - Molecular-Dynamics of $\mathrm{N}$ Alkanes, J Comput Phys. 23, 327-341.

95. Darden, T., York, D. \& Pedersen, L. (1993) Particle mesh Ewald: An N.log(N) method for Ewald sums in large systems, $J$ Chem Phys. 98, 10089-10092.

96. Quigley, D. \& Probert, M. I. (2004) Langevin dynamics in constant pressure extended systems, J Chem Phys. 120, 11432-41.

97. Berger, O., Edholm, O. \& Jahnig, F. (1997) Molecular dynamics simulations of a fluid bilayer of dipalmitoylphosphatidylcholine at full hydration, constant pressure, and constant temperature, Biophys J. 72, 2002-2013. 
1200

1201

98. Berendsen, H. J. C., Postma, J. P. M., van Gunsteren, W. F., DiNola, A. \& Haak, J. R. (1984) Molecular dynamics with coupling to an external bath, J Chem Phys. 81, 3684-3690.

99. Roe, D. R. \& Cheatham, T. E., 3rd (2013) PTRAJ and CPPTRAJ: Software for Processing and Analysis of Molecular Dynamics Trajectory Data, J Chem Theory Comput. 9, 3084-95.

100. Schrodinger, LLC (2015) The PyMOL Molecular Graphics System, Version 1.8

101. Humphrey, W., Dalke, A. \& Schulten, K. (1996) VMD: Visual molecular dynamics, J. Molec. Graphics. 14, 33-38.

102. Paloncyova, M., Berka, K. \& Otyepka, M. (2012) Convergence of Free Energy Profile of Coumarin in Lipid Bilayer, J Chem Theory Comput. 8, 1200-1211.

103. Marrink, S.-J., Berger, O., Tieleman, P. \& Jähnig, F. (1998) Adhesion Forces of Lipids in a Phospholipid Membrane Studied by Molecular Dynamics Simulations, Biophys J. 74, 931943.

104. Chen, P. C. \& Kuyucak, S. (2011) Accurate determination of the binding free energy for KcsA-charybdotoxin complex from the potential of mean force calculations with restraints, Biophys J. 100, 2466-74.

105. Schymkowitz, J., Borg, J., Stricher, F., Nys, R., Rousseau, F. \& Serrano, L. (2005) The FoldX web server: an online force field, Nucleic Acids Res. 33, W382-8.

106. Guerois, R., Nielsen, J. E. \& Serrano, L. (2002) Predicting changes in the stability of proteins and protein complexes: A study of more than 1000 mutations, J Mol Biol. 320, 369387.

107. Tokuriki, N., Stricher, F., Schymkowitz, J., Serrano, L. \& Tawfik, D. S. (2007) The stability effects of protein mutations appear to be universally distributed, $J$ Mol Biol. 369, 1318-32.

108. Li, M. Z. \& Elledge, S. J. (2007) Harnessing homologous recombination in vitro to generate recombinant DNA via SLIC, Nat. Methods 4, 251-6.

109. Bleffert, F., Granzin, J., Gohlke, H., Batra-Safferling, R., Jaeger, K.-E. \& Kovacic, F. (2019) Pseudomonas aeruginosa esterase PA2949, a bacterial homolog of the human membrane esterase ABHD6: expression, purification and crystallization, Acta Crystallogr F. 75, 270-277.

110. Laemmli, U. K. (1970) Cleavage of structural proteins during the assembly of the head of bacteriophage T4, Nature 227, 680-685.

111. Jaeger, K. E. \& Kovacic, F. (2014) Determination of lipolytic enzyme activities, Methods Mol Biol. 1149, 111-134.

112. Kovacic, F., Granzin, J., Wilhelm, S., Kojic-Prodic, B., Batra-Safferling, R. \& Jaeger, K. E. (2013) Structural and functional characterisation of TesA - a novel lysophospholipase A from Pseudomonas aeruginosa, PLoS One. 8, e69125. 
113. Viegas, A., Dollinger, P., Verma, N., Kubiak, J., Viennet, T., Seidel, C. A. M., Gohlke, H., Etzkorn, M., Kovacic, F. \& Jaeger, K.-E. (2020) Structural and dynamic insights revealing how lipase binding domain MD1 of Pseudomonas aeruginosa foldase affects lipase activation, Sci Rep. 10, 3578.

114. Bayly, C. I., Cieplak, P., Cornell, W. \& Kollman, P. A. (1993) A well-behaved electrostatic potential based method using charge restraints for deriving atomic charges: the RESP model, J Phys Chem. 97, 10269-10280.

115. Wang, J., Wang, W., Kollman, P. A. \& Case, D. A. (2006) Automatic atom type and bond type perception in molecular mechanical calculations, J Mol Graph Model. 25, 247-60.

116. Frisch, M. J., Trucks, G. W., Schlegel, H. B., Scuseria, G. E., Robb, M. A., Cheeseman, J. R., Scalmani, G., Barone, V., Petersson, G. A., Nakatsuji, H., Li, X., Caricato, M., Marenich, A. V., Bloino, J., Janesko, B. G., Gomperts, R., Mennucci, B., Hratchian, H. P., Ortiz, J. V., Izmaylov, A. F., Sonnenberg, J. L., Williams-Young, D., Ding, F., Lipparini, F., Egidi, F., Goings, J., Peng, B., Petrone, A., Henderson, T., Ranasinghe, D., Zakrzewski, V. G., Gao, J., Rega, N., Zheng, G., Liang, W., Hada, M., Ehara, M., Toyota, K., Fukuda, R., Hasegawa, J., Ishida, M., Nakajima, T., Honda, Y., Kitao, O., Nakai, H., Vreven, T., Throssell, K., Montgomery Jr., J. A., Peralta, J. E., Ogliaro, F., Bearpark, M. J., Heyd, J. J., Brothers, E. N., Kudin, K. N., Staroverov, V. N., Keith, T. A., Kobayashi, R., Normand, J., Raghavachari, K., Rendell, A. P., Burant, J. C., lyengar, S. S., Tomasi, J., Cossi, M., Millam, J. M., Klene, M., Adamo, C., Cammi, R., Ochterski, J. W., Martin, R. L., Morokuma, K., Farkas, O., Foresman, J. B. \& Fox, D. J. (2016) Gaussian 16 Revision A.03 in Gaussian, Inc., Wallingford, CT, .

117. Wang, J., Wolf, R. M., Caldwell, J. W., Kollman, P. A. \& Case, D. A. (2004) Development and testing of a general amber force field, $J$ Comput Chem. 25, 1157-74. 